August 3, 2020

\title{
A genome compendium reveals diverse metabolic adaptations of Antarctic soil microorganisms
}

Maximiliano Ortiz ${ }^{1}$, Pok Man Leung ${ }^{2}$ * , Guy Shelley ${ }^{3}$, Marc W. Van Goethem ${ }^{1,4}$, Sean K. Bay ${ }^{2}$, Karen Jordaan ${ }^{1,5}$, Surendra Vikram ${ }^{1}$, Ian D. Hogg ${ }^{1,7,8}$, Thulani P. Makhalanyane ${ }^{1}$, Steven L. Chown ${ }^{6}$, Rhys Grinter ${ }^{2}$, Don A. Cowan ${ }^{1}$ *, Chris Greening ${ }^{2,3}$ *

${ }^{1}$ Centre for Microbial Ecology and Genomics, Department of Biochemistry, Genetics and Microbiology, University of Pretoria, Hatfield, Pretoria 0002, South Africa

2 Department of Microbiology, Monash Biomedicine Discovery Institute, Clayton, VIC 3800, Australia

${ }^{3}$ School of Biological Sciences, Monash University, Clayton, VIC 3800, Australia

4 Environmental Genomics and Systems Biology Division, Lawrence Berkeley National Laboratory, Berkeley, California, USA

5 Departamento de Genética Molecular y Microbiología, Facultad de Ciencias Biológicas, Pontificia Universidad Católica de Chile, Alameda 340, Santiago

6 Securing Antarctica's Environmental Future, School of Biological Sciences, Monash University, Clayton, VIC 3800, Australia

${ }^{7}$ School of Science, University of Waikato, Hamilton 3240, New Zealand

8 Polar Knowledge Canada, Canadian High Arctic Research Station, Cambridge Bay, NU X0B 0C0, Canada

\# These authors contributed equally to this work.

* Correspondence may be addressed to:

A/Prof Chris Greening (chris.greening@monash.edu)

Prof Don A. Cowan (don.cowan@up.ac.za)

Pok Man Leung (pok.leung@monash.edu) 


\section{Abstract}

2 A surprising diversity and abundance of microorganisms resides in the cold desert

3 soils of Antarctica. The metabolic processes that sustain them, however, are poorly

4 understood. In this study, we used metagenomic and biogeochemical approaches to

5 study the microbial communities in 16 physicochemically diverse mountainous and

6 glacial soils from remote sites in South Victoria Land, north of the Mackay Glacier.

7 We assembled 451 metagenome-assembled genomes from 18 bacterial and

8 archaeal phyla, constituting the largest resource of Antarctic soil microbial genomes

9 to date. The most abundant and prevalent microorganisms are metabolically

10 versatile aerobes that use atmospheric hydrogen and carbon monoxide to meet

11 energy, carbon, and, through metabolic water production, hydration needs.

12 Phylogenetic analysis and structural modelling infer that bacteria from nine phyla can

13 scavenge atmospheric hydrogen using a previously unreported enzyme family, the

14 group 1 [ [NiFe]-hydrogenases. Consistently, gas chromatography measurements

15 confirmed most soils rapidly consume atmospheric hydrogen and carbon monoxide,

16 and provide the first experimental evidence of methane oxidation in non-maritime

17 Antarctica. We also recovered genomes of microorganisms capable of oxidizing

18 other inorganic compounds, including nitrogen, sulfur, and iron compounds, as well

19 as harvesting solar energy via photosystems and novel microbial rhodopsins.

20 Bacterial lineages defined by symbiotic lifestyles, including Patescibacteria,

21 Chlamydiae, and predatory Bdellovibrionota, were also surprisingly abundant. We

22 conclude that the dominant microorganisms in Antarctic soils adopt mixotrophic

23 strategies for energy and sometimes carbon acquisition, though they co-exist with

24 diverse bacteria and archaea that adopt more specialist lifestyles. These

25 unprecedented insights and associated genome compendium will inform efforts to

26 protect biodiversity in this continent. 


\section{Introduction}

28 Continental Antarctica is a relatively pristine but oligotrophic wilderness ${ }^{1}$. Terrestrial

29 life on the continent is adapted to extremely low temperatures, low water

30 bioavailability, highly limited organic carbon and nitrogen, salt accumulation and

31 seasonal light/dark periodicity ${ }^{2-4}$. These cumulative pressures exclude most

32 macroscopic fauna and flora, and instead microorganisms constitute most of the

33 continent's biodiversity and biomass ${ }^{5}$. While historical observational surveys

34 indicated that few microorganisms existed in terrestrial Antarctica, subsequent

35 molecular studies have uncovered rich and abundant microbial communities, especially in the continent's ice-free regions ${ }^{6-10}$. Antarctic soil communities are comparable to mesophilic soils at the phylum level, with Actinobacteriota, Acidobacteriota, Chloroflexota and Proteobacteria often predominant ${ }^{2,8,9,11,12}$. These communities are highly specialised at lower taxonomic levels ${ }^{7,8}$, however, and have unique functional traits ${ }^{11,13}$. Complementary culture-based studies have also isolated a growing number of taxa from the continent, although from relatively few phyla $^{14-17}$. Most community members are assumed to be extremely slow-growing or adopt dormant states to adapt to the physicochemical conditions of the continent ${ }^{18}$. In turn, the formation of a microbial 'seed bank' may provide a means to maintain biodiversity ${ }^{19,20}$.

An enduring question is what metabolic strategies enable soil microorganisms to meet energy and carbon needs on this continent ${ }^{2}$. Even in dormant states, cells still require a net energy input to maintain cellular integrity, repair damaged macromolecules, and generate a basal membrane potential ${ }^{21,22}$. Conventionally it was thought that Cyanobacteria and microalgae are the major primary producers in Antarctic soils and that they produce the organic carbon to sustain organoheterotrophic bacteria ${ }^{2,11}$. However, oxygenic photoautotrophs are typically in low abundance ( $<1 \%$ of total bacterial community) outside lithic niches ${ }^{11,23}$ and hence are unlikely to produce sufficient organic carbon to sustain the energy and carbon needs of the dominant community members. More recently, some Antarctic soil bacteria were shown to conserve energy and acquire carbon independently of

57 photoautotrophs ${ }^{12}$. Genome-centric metagenomic studies have revealed that 58 bacteria from several phyla, including Actinobacteriota, consume molecular 
hydrogen $\left(\mathrm{H}_{2}\right)$ and carbon monoxide $(\mathrm{CO})$ from the atmosphere. By liberating electrons from these ubiquitous and diffusible trace gases, these bacteria sustain aerobic respiration and fix carbon even when preferred organic substrates are limiting ${ }^{12,24}$. However, given the relatively few metagenome-assembled genomes (MAGs) recovered (21) and limited geographical scope of this previous study ${ }^{12}$, it is unknown whether trace gas oxidation is a widespread strategy among Antarctic bacteria. Several molecular and biogeochemical studies have detected signatures of carbon fixation through the Calvin-Benson-Bassham (CBB) cycle within the continent, though it is unclear whether this originates through activities of photoautotrophs or lithoautotrophs ${ }^{12,13,25-27}$. Molecular evidence also suggests that some Antarctic soil bacteria can also conserve energy through other means, including methanotrophy, nitrification, and rhodopsin-based light harvesting ${ }^{12,13,16,28-}$ 30 .

Here we build on these initial findings to develop a holistic genome-resolved understanding of the metabolic capabilities of Antarctic soil microorganisms. We profiled 16 soils with distinct physicochemical properties from the Mackay Glacier region, a cold hyper-arid ice-free region to the north of the McMurdo Dry Valleys that comprises approximately $15 \%\left(\sim 4,800 \mathrm{~km}^{2}\right)$ of the ice-free regions on the continent. Soil microbial communities in this region are adapted to average annual temperatures of $-20^{\circ} \mathrm{C}$ and annual precipitation below $50 \mathrm{~mm}{ }^{31,32}$, as well as profound limitation for organic carbon $(\sim 0.1 \%)$ and nitrogen $(\sim 0.02 \%){ }^{33}$. Through deep metagenomic sequencing, we generated a resource of 451 metagenomeassembled genomes, covering all major microbial lineages in the region. We confirmed that the most abundant bacteria in the region are mixotrophs that scavenge atmospheric trace gases, and substantiated these findings with biogeochemical assays confirming rapid gas consumption and phylogenetic analyses revealing a novel hydrogenase family. These findings lend strong support to the recent hypothesis that survival in desert soils depends on continual harvesting of alternative energy sources ${ }^{18}$. Nevertheless, these metabolically versatile bacteria co-exist with microorganisms that adopt a wide range of other nutritional and ecological strategies, including apparent obligate parasites and predators. Altogether, Antarctic soils appear to harbour much more compositionally rich and functionally complex microbial life than previously assumed. 


\section{Results and Discussion}

93 Genome-resolved metagenomics reveals phylogenetically diverse bacteria coexist across the Mackay Glacier region

95 We analyzed surface soils from sixteen glacial and mountainous sites sampled 96 across the Mackay Glacier region of South Victoria Land. Physicochemical analysis confirmed that the soils varied in key properties (e.g. $\mathrm{pH}$, salinity, micronutrients, texture), but in common with previously characterized soils from continental Antarctic regions $^{8,34,35}$, all had exceptionally low organic carbon content (0.02 - 0.25\%) (Table S1). These soils nevertheless supported moderately abundant bacterial and archaeal communities $\left(1.7 \times 10^{6}\right.$ to $2.7 \times 10^{7} 16 \mathrm{~S}$ rRNA gene copies per gram soil wet weight) (Figure 1a). Based on high-resolution 16S rRNA amplicon sequencing ${ }^{36}$ (Figure S1a \& S1b), observed richness (832 \pm 258) and Shannon index (5.27 \pm 0.31) were high in most samples, implying diverse community members co-exist in these soils (Figure 1c; Figure S1d). Beta diversity analysis confirmed microbial communities diverge between sampled regions and with geographic distance (Figure 1d; Figure S1e).

To determine the community composition of the samples, we retrieved and classified shotgun metagenomic reads of the universal single-copy ribosomal protein gene rp/P (Table S2). The dominant community members were from bacterial phyla known to predominate in soil ecosystems ${ }^{37,38}$. Actinobacteriota, Proteobacteria,

112 Acidobacteriota, Chloroflexota, Gemmatimonadota, Verrucomicrobiota and 113 Bacteroidota were particularly abundant (Figure 1b), in agreement with other 114 Antarctic surveys ${ }^{2,18}$. Cyanobacteria were scarce in most soils except for Pegtop Mountain and Cliff Nunatak, accounting for an average of $0.50 \%$ in the soil communities. Likewise, Archaea were minor members of this ecosystem (av. $0.88 \%$ ) and mainly comprised the ammonia-oxidizing order Nitrososphaerales (Figure 1b).

118 More surprisingly, bacterial phyla that predominantly adopt a predatory 119 (Bdellovibrionota) ${ }^{39}$, intracellular parasitic (Dependentiae and Verrucomicrobiota_A / 120 Chlamydiae) ${ }^{40,41}$ or obligately symbiotic (Patescibacteria) ${ }^{42,43}$ lifestyle were 121 prevalent and sometimes highly abundant, for example together comprising $17 \%$ of the community at Mount Murray. This suggests that a range of symbiotic interactions 
occur in these communities. These dominant and rare phyla were also detected by 16S rRNA gene sequencing (Figure S1c; Table S3).

These inferences on the composition and metabolic capabilities of the microbial communities were supported by genome-resolved analysis. From the 99.5 gigabases of sequencing data (Table S4), we reconstructed a non-redundant set of 101 high-quality and 350 medium-quality ${ }^{44}$ metagenome-assembled genomes (MAGs). The recovered genomes span 18 different phyla, the relative composition of which reflects the community structure patterns observed in the rpIP and 16S rRNA analysis (Figure 1b). In turn, they capture all major microbial lineages (present at $>1 \%$ relative abundance across all samples) and map to an average of $26 \%$ of reads in each metagenome (Table S5). To the best of our knowledge, this represents the largest sequencing effort and most extensive genomic resource reported from terrestrial Antarctica to date.

\section{Most abundant lineages encode enzymes supporting trace gas oxidation, including a novel family of [NiFe]-hydrogenases}

We sought to understand which metabolic strategies support the numerous bacteria in these hyper-oligotrophic soils. We profiled the distribution and affiliation of 52 conserved marker genes representing different energy conservation and carbon acquisition pathways in both the metagenomic short reads (Table S6) and MAGs (Table S5). In line with expectations, almost all community members encoded genes for aerobic organotrophic respiration (CoxA, NuoF, SdhA, AtpA) (Figure 2), whereas capacity for anaerobic respiration and fermentation was low (Figure S2). In addition to formate dehydrogenase, the other most abundant markers were the catalytic subunits of [NiFe]-hydrogenases (present in average of $90 \%$ community members), form I carbon monoxide dehydrogenases (32\%), and RuBisCO (27\%) (Figure 2). Phylogenetic analysis revealed that most binned sequences of these enzymes were most closely related to clades that support atmospheric $\mathrm{H}_{2}$ oxidation ${ }^{45-49}$ (Figure 3a), atmospheric CO oxidation ${ }^{12,50-53}$ (Figure S3), and chemosynthetic $\mathrm{CO}_{2}$ fixation 12,54-56 (Figure S4). Recent pure culture studies have shown that energy liberated by atmospheric $\mathrm{H}_{2}$ and $\mathrm{CO}$ oxidation supports bacterial persistence during carbon starvation and, in some cases, mixotrophic growth 52,57-62. Thus, the ability of 
155

156

157

bacteria to harvest these trace gases may confer a major selective advantage in the carbon-depleted soils of Antarctica. Moreover, in extension of findings made in the Windmill Islands region ${ }^{12}$, over a quarter of the community may fix carbon via the CBB cycle, providing a mean to generate biomass independently of photoautotrophy.

Genes for trace gas oxidation were present in the most abundant and widespread community members. Uptake hydrogenases were encoded by MAGs affiliating with nine bacterial phyla (Figure 2 \& 3a), including the seven dominant soil phyla (Figure 1), whereas $\mathrm{CO}$ dehydrogenases were confined to Actinobacteriota and Chloroflexota (Figure S3). Indeed, 17 of the 20 most abundant Actinobacteriota and Chloroflexota MAGs encoded one or both enzymes (Table S5). Remarkably, the CBB pathway (Figure S4; Table S7) frequently co-occurs with hydrogenases (64\%) and $\mathrm{CO}$ dehydrogenase (25\%) in MAGs (Figure 2; Table S5), potentially enabling hydrogenotrophic, carboxydotrophic or mixotrophic growth. This association was especially pronounced in the uncultivated classes Ellin6529 (Chloroflexota) and UBA4738 (Actinobacteriota) (Table S6), which respectively comprise an average of $5.1 \%$ and $0.9 \%$ (maximum of $12.3 \%$ and $2.4 \%$ ) of the communities across the region (Table S2). These classes are predicted to couple atmospheric $\mathrm{H}_{2}$ and $\mathrm{CO}$ oxidation to fix carbon via their respective type IC and IE RuBisCO enzymes (Figure S4; Table S7). These traits in turn may contribute to their unexpectedly high relative abundance in Antarctica as well as other oligotrophic soils ${ }^{15,63-66}$. Indeed, given their abundance in the community and genetic potential for atmospheric chemosynthesis 12,24, we hypothesize that both classes are major Antarctic primary producers. We propose replacing the placeholder names UBA4738 with Candidatus Aridivitia (arid Actinobacteriota class; based on high-quality type MAG MGR_bin238, 'Candidatus Aridivita willemsiae') and Ellin6529 with Candidatus Edaphomicrobia (edaphic Chloroflexota class; based on high-quality type MAG MGR_130 'Candidatus Edaphomicrobium janssenii') (Etymological Information), as per recent taxonomic recommendations ${ }^{67,68}$.

Most microorganisms in the Mackay Glacier region encoded a novel hydrogenase family (Figure 2). We generated a maximum-likelihood tree of the conserved catalytic subunits of group 1 [NiFe] hydrogenases using amino acid sequences retrieved from 176 MAGs. All hydrogenase sequences form two major and tremendously diverse lineages that share less than $40 \%$ sequence identity with each 
188 other and were supported by robust bootstrapping (Figure 3a). One branch is associated with characterized group $1 \mathrm{~h}$ [NiFe] hydrogenases from multiple bacterial isolates $45-47,51,61$. The other forms a novel cluster, herein the group 1 [ [NiFe]hydrogenase, which includes the previously unreported hydrogenases of McMurdo Dry Valleys isolate Hymenobacter roseosalivarius ${ }^{69}$ and several other recently sequenced isolates. Group $1 \mathrm{l}$ is the prevailing hydrogenase family within the Mackay Glacier region, with an estimated abundance 2.3 times higher than group $1 \mathrm{~h}$ (Table S5), and is encoded by all nine hydrogenase-bearing phyla and the two candidate classes. As elaborated in Supplementary Note 1, structural modelling shows that this enzyme shares common structural features with previously characterized group 1h [NiFe]-hydrogenase ${ }^{70,71}$, but contains large sequence insertions and a key substitution in a residue ligating the proximal iron-sulfur cluster. Even more strikingly, the genes encoding this hydrogenase often have an unusual arrangement (Figure S5; Table S7), with five open reading frames predicted to encode small transmembrane proteins separating the small and large core structural subunits. On this basis, we predict that this enzyme is a bona fide high-affinity membraneassociated hydrogenase that relays electrons derived from atmospheric $\mathrm{H}_{2}$ through the respiratory chain. The broad distribution and predominance of this hydrogenase suggests it is the primary mediator of $\mathrm{H}_{2}$ oxidation in these soils. Moreover, given the strong positive correlation between this hydrogenase and RuBisCO based on the MAGs and metagenomic short reads $\left(R^{2}=0.68, p=0.002\right)$ (Figure S6; Table S9), it is likely that electrons yielded by this enzyme support carbon fixation either through direct transfer or reverse electron flow.

\section{Trace gas consumption occurs at sufficient rates to meet energy needs and support hydration of Mackay Glacier region bacteria}

Our metagenomic analyses suggest that the most abundant soil bacteria across the Mackay Glacier region conserve energy and fix carbon by oxidizing atmospheric $\mathrm{H}_{2}$ and CO. To test whether soil communities mediate these activities, we set up soil microcosms in which ambient air headspaces were amended with 10 parts per million (ppmv) of these gases and used high-sensitivity gas chromatography to measure their consumption over time. In line with predictions, $\mathrm{H}_{2}$ was oxidized by 
soils from all sixteen sites and all but three soils consumed CO (Figure 4a). Of these, all soils except Pegtop Mountain consumed $\mathrm{H}_{2}$ to below atmospheric concentrations (0.53 ppmv) ${ }^{72}$ and ten soils consumed atmospheric CO (0.09 ppmv) 73 during the timecourse of our experiments (Figure S7). These sub-atmospheric thresholds confirm that these microbial communities can harvest energy from the atmosphere, a virtually unlimited source of diffusive and energy-rich reduced gases ${ }^{74,75}$. The average rate of atmospheric $\mathrm{H}_{2}$ oxidation (135 $\mathrm{pmol} \mathrm{hr}^{-1} \mathrm{~g}_{\text {soil ww }}{ }^{-1}$ ) was much faster than for atmospheric CO oxidation (0.60 pmol hr${ }^{-1} \mathrm{~g}_{\text {soil }} \mathrm{ww}^{-1}$ ) (Table S8). This finding, together with the higher abundance of putative $\mathrm{H}_{2}$ oxidizers in the soil communities (Figure 2), suggests that atmospheric $\mathrm{H}_{2}$ is likely to be the predominant energy source sustaining these communities. As elaborated in Supplementary Note 2, considerable variations in bulk and normalized oxidation rates were measured for both gases, which was significantly correlated with several measured physicochemical variables (Figure S6; Table S9).

Cell-specific rates were calculated by normalizing bulk rates against soil microbial abundance and the proportion of trace gas oxidizers. Cell-specific atmospheric $\mathrm{H}_{2}$ oxidation rates were high (av. $1.1 \times 10^{-7} \mathrm{nmol} \mathrm{hr}^{-1}$ cell $^{-1}$ ) and approximately two orders of magnitude higher than those of CO (av. $1.3 \times 10^{-9} \mathrm{nmol} \mathrm{hr}^{-1} \mathrm{cell}^{-1}$ ) (Figure 4b). In line with our findings in the Windmill Islands region ${ }^{12}$, this rate of atmospheric $\mathrm{H}_{2}$ consumption exceeds the theoretical maintenance requirements of trace gas oxidizers at the temperature tested $\left(10^{\circ} \mathrm{C}\right)$ and is sufficient to support some growth 76-78. It should also be noted that metabolic water is the major end-product of the aerobic respiration of atmospheric $\mathrm{H}_{2}\left(2 \mathrm{H}_{2}+\mathrm{O}_{2} \rightarrow 2 \mathrm{H}_{2} \mathrm{O}\right)$. Given the reported cytosolic orientation of high-affinity hydrogenases and terminal oxidases ${ }^{62}$, the water produced would be retained in the cytosol, including as a solvent for macromolecules. Thus, trace gas oxidation may be a simple, but hitherto overlooked, mechanism for microorganisms to stay hydrated in the hyper-arid deserts of Antarctica. Based on cell-specific rates of atmospheric $\mathrm{H}_{2}$ oxidation, a theoretical average of 1.1 million water molecules would be produced per cell each minute. For a cell with an expected $1 \mu^{3}$ volume and $70 \%$ water content ${ }^{79,80}$, such production rates would be sufficient to replace all cellular water over a 15-day period (Table S8). We therefore propose that the metabolic water continuously generated by trace 
252

253

254

255

256

257

258

259

260

261

262

gas oxidation is a quantitatively significant source of hydration in this environment with minimal precipitation ${ }^{32}$.

Metabolically constrained phototrophs, lithotrophs, and organotrophs co-exist with versatile mixotrophs in Antarctic soils

While the most abundant taxa in the Mackay Glacier ecotone appear to be versatile mixotrophs, the genome compendium revealed that these ecosystems also harbor diverse bacteria and archaea with specialist strategies for energy and carbon acquisition. Multiple chemolithoautotrophs were present, including those capable of oxidizing the trace amounts of ammonium, sulfur and iron detected in the soils (Table S1). Ammonium and nitrite oxidizers comprised an average of $2.9 \%$ and $1.0 \%$ of the communities, but together comprised $23 \%$ and $15 \%$ of the community in Mount Seuss 6 and Benson Glacier samples, respectively (Figure 2; Table S6). Phylogenetic analysis confirmed that Nitrososphaerales (archaea) and Burkholderiales (bacteria) were the dominant ammonium oxidizers (Figure S8), in line with previous reports for McMurdo Dry Valley soils ${ }^{28}$, whereas Nitrospirota were the main nitrite oxidizers (Figure S9). These nitrifiers also respectively encoded the signature enzymes to fix carbon through the archaeal 4-hydroxybutyrate cycle (Figure S10), proteobacterial CBB cycle (Figure S4), and nitrospiral reverse tricarboxylic acid cycle (Figure S11), suggesting that multiple chemosynthetic primary production strategies sustain biodiversity in these oligotrophic soils. The marker genes for sulfide and thiosulfate oxidation (Sqr, FCC, SoxB) were each encoded by 1 - 4\% of community members in most soils (Figure 2; Table S5), including multiple Burkholderiales MAGs and several other lineages (Figure S12, $\mathbf{S 1 3}, \mathbf{S 1 4})$. The genes to oxidize ferrous iron via the $c$-type cytochrome Cyc2 were widespread in Mount Seuss 6 (4.7\%) and Cliff Nunatak samples (7.3\%), and present in select MAGs from five major phyla (Figure S15). Thus, atmospheric and edaphic inorganic compounds alike are major energy sources for Antarctic soil communities, although their relative importance varies across the physicochemically diverse soils from the region.

Our metagenomic analysis suggests that light energy supports few photoautotrophs, but numerous photoheterotrophs, in the region. Reflecting cyanobacterial 
284 distributions across the region (Figure 1b), photosystems associated with oxygenic 285 photosynthesis were encoded by few community members except in the Pegtop 286 Mountain and Cliff Nunatak samples (Figure 2). Some photosystem II sequences 287 affiliated with proteobacterial anoxygenic phototrophs were also detected (Figure 288 S16). In contrast, energy-converting microbial rhodopsins were prevalent and 289 abundant across the region (Figure 2). These light-powered proton pumps are well290 characterized for their role in energy conservation in marine and freshwater 291 ecosystems ${ }^{81-85}$, though have been scarcely studied in desert environments ${ }^{86}$. As 292 outlined by our 'continual energy harvesting hypothesis', sunlight (in common with 293 atmospheric trace gases) is a relatively dependable energy source and hence 294 lineages that harvest it may have a selective advantage in energy-poor desert soils 295 18. In line with this theory, putative energy-converting rhodopsins were present in several of the most dominant orders of Actinobacteriota and Chloroflexota in these soils (Table S5). They were also present in both cyanobacterial MAGs, thereby providing a means for photoautotrophs to conserve energy when water for oxygenic photosynthesis is limiting (Figure S17). Phylogenetic analysis confirmed the binned and unbinned sequences fell into diverse clades (Figure S17), including two novel clades that were most closely related ( $<50 \%$ sequence identity) to the biochemically characterized energy-converting rhodopsins of halophilic archaea (bacteriorhodopsins) ${ }^{87}$ and Pantoea species (pantorhodopsins) ${ }^{88}$.

Twenty metagenome-assembled genomes were also recovered for the phyla known to adopt obligately symbiotic lifestyles, namely Patescibacteria, Chlamydiae, Dependentiae, and Bdellovibrionota (Table S5). All four phyla appear to be obligate organoheterotrophs that lack alternative pathways for energy conservation or carbon acquisition (Figure 2). Based on previous reports, all characterized Bdellovibrionota predate bacterial species ${ }^{39}$, whereas Chlamydiae and Dependentiae are likely to be parasites of protist or arthropod species ${ }^{40,41,89}$ such as populations of springtails (Collembola) identified within the same sampling area ${ }^{90}$. Signature genes associated with the symbiotic lifestyles of each MAG were detected, for example host-targeted peptidoglycan metalloendopeptidases and self-protection proteins that Bdellovibrionota uses to invade cells of bacterial prey ${ }^{91,92}$, as well as ankyrin repeat and WD40 repeat proteins implicated in modulation of eukaryotic hosts by Dependentiae ${ }^{41,89}$ (Table S5). Also in line with an obligately symbiotic lifestyle, 
several lineages have ultra-small genomes when adjusted for completeness, namely the eight Patescibacteria MAGs (av. 1.3 Mbp), three Dependentiae MAGs (av. 1.8 Mbp), and a Rickettsiaceae MAG (1.3 Mbp) (Table S5), and are predicted to be auxotrophic for multiple amino acids. Building on the discovery of unexpected symbionts in Antarctic lakes ${ }^{93,94}$, to our knowledge this is the first report that microbial parasitism is a major ecological strategy in terrestrial Antarctica. We also reveal oxic niches for phyla such as Patescibacteria that have, until now, primarily been studied in anoxic ecosystems ${ }^{42,95,96}$.

Finally, we obtained genomic and biogeochemical evidence that atmospheric methane oxidation occurs on non-maritime Antarctic soils. Based on methane monooxygenase levels in short reads, aerobic methanotrophs are members of the rare biosphere in most of the sampled Antarctic soils, but are present in very high levels in three soils, including Mount Seuss 5 (9.4\%) (Figure 2; Table S5). Concordantly, two of these soils oxidized methane at high cell-specific rates to subatmospheric levels during microcosm incubations (Figure 4; Figure S7). Genomeresolved analysis suggested that this activity is primarily mediated by a single bacterial species within the gammaproteobacterial order UBA7966, which encodes a particulate methane monooxygenase clustering with sequences from the atmospheric methane-oxidizing clade USCy (Figure S18). While this bacterium has a restricted distribution, based on read mapping, it is among the most abundant single taxon across the entire region (Table S5). Thus, by adopting a relatively specialist lifestyle dependent on assimilating a widely available but catalytically demanding atmospheric substrate, this bacterium fills a distinct ecological niche. Importantly, although methanotroph genomes have previously been reported in Antarctic soils ${ }^{12,30}$, this is the first experimental report that such bacteria are biogeochemically

active. 


\section{Conclusions}

344 Altogether, these results demonstrate a remarkable diversity of both microbial 345 lineages and metabolic strategies in the resource-poor soils of Antarctica. The most

346 abundant and prevalent bacterial lineages in Antarctic soils appear to be free-living 347 mixotrophs capable of meeting carbon, energy, and even hydration needs from 348 atmospheric trace gases, i.e. 'living on air' ${ }^{97}$. Several bacteria and archaea also 349 achieve high abundances in specific soils through more specialist strategies, 350 spanning atmospheric methanotrophy, oxygenic photosynthesis and lithoautotrophic 351 growth on trace edaphic substrates. This environment in turn has selected for a 352 range of as-yet-uncultivated bacterial lineages (e.g. Ca. Edaphomicrobia and $\mathrm{Ca}$. 353 Aridivitia) and previously unreported gene families (e.g. encoding group 11 [NiFe]354 hydrogenases and potential microbial rhodopsins). Also, surprisingly, a significant 355 minority of community members gain resources through parasitism or predation of 356 microorganisms. Through this combination of strategies, both free-living and 357 symbiotic microorganisms can achieve stable niches in a polyextreme environment.

358 Additionally, the wealth of metagenomic sequencing data and 451 draft genomes 359 generated by this study provides a valuable resource for two major areas of 360 endeavor. First, these datasets support fundamental research and potentially inform 361 decisions to secure Antarctica's environmental future, given forecasts of changing 362 temperature and water availability ${ }^{98-100}$. Thus, in line with one of the six priorities for 363 Antarctic science ${ }^{101}$, this resource will provide insights into how life has evolved and 364 adapted on this microbially-dominated continent, and in turn may respond to climate 365 changes. Secondly, these findings also contribute to considerations of what 366 processes may sustain life on other cold, dry planets such as Mars. Antarctica has 367 long been considered a potential analogue for life elsewhere in the solar system ${ }^{102}$. 368 Our work brings that picture into sharper resolution. 


\section{Materials and Methods}

\section{Soil physicochemical analysis}

This study used mineral soils previously sampled from 16 glacier- or mountainassociated sites in the Mackay Glacier region, South Victoria Land, Antarctica during January 2015 as previously described ${ }^{33,35}$. In brief, $50 \mathrm{~g}$ of surface soil (depth: 0 - 5 $\mathrm{cm}$ ) at each location was collected from an approximately $1 \mathrm{~m}^{2}$ area and stored in sterile $50 \mathrm{ml}$ polypropylene Falcon tubes (Grenier, Bio-One) aseptically. During storage and transportation to University of Pretoria, samples were kept at $-80^{\circ} \mathrm{C}$. They were later shipped to Monash University's quarantine approved facilities for further experiments. Details of soil samples can be found in Table S1. Prior to physicochemical measurements, approximately $35 \mathrm{~g}$ of soil of individual sample was aliquoted. Soil aliquots were treated with gamma irradiation at $50 \mathrm{kGy}$ (Steritech Pty Ltd Victoria, Australia) for compliance with Department of Agriculture, Water and the Environment's quarantine good regulations. They were subsequently shipped to the Environmental Analysis Laboratory (EAL), Southern Cross University, Australia for physicochemical analyses in accordance with ISO/IEC 17025 standard procedures. Physicochemical parameters analysed included: basic soil colour and texture; $\mathrm{pH}$ and electrical conductivity (1:5 water); moisture content; total carbon, nitrogen, organic carbon, and organic matter; available calcium, magnesium, potassium, ammonium, nitrate, phosphate, sulfur; exchangeable sodium, potassium, calcium, magnesium, hydrogen, and aluminium; cation exchange capacity; Bray I, Bray II, and Cowell phosphorus; and available micronutrients zinc, manganese, iron, copper, boron, and silicon. These data are summarised in Table S1.

\section{Shotgun metagenome sequencing, assembly and binning}

Community DNA for metagenomic sequencing was extracted from $0.5 \mathrm{~g}$ of soil using the FastDNA SPIN Kit for soil (MP Biomedicals) according to the manufacturer's instructions. An extraction blank control was included. Metagenomic shotgun libraries were prepared using the Nextera XT DNA Sample Preparation Kit (Illumina Inc., San Diego, CA, USA) and subject to paired-end sequencing ( $2 \times 150 \mathrm{bp}$ ) on an Illumina NextSeq500 platform at the Australian Centre for Ecogenomics (ACE), University of Queensland. Sequencing yielded 356,941,066 read pairs across the sixteen soil metagenomes and 556 read pairs for the negative control (Table S4), 
402 indicating a minimal level of contamination from DNA extraction and sequencing 403 processes. Raw metagenomic sequences were subjected to quality filtering using 404 the BBDuk function of the BBTools v38.80 (https://sourceforge.net/projects/bbmap/); 405 contaminating adapters ( $\mathrm{k}$-mer size of 23 and hamming distance of 1), PhiX 406 sequences (k-mer size of 31 and hamming distance of 1), and bases from 3' ends 407 with a Phred score below 20 were trimmed. After removing resultant reads with 408 lengths shorter than 50 bp, 93\% high-quality read pairs were retained for 409 downstream analysis. Metagenomic reads from each sample were assembled 410 individually with metaSPAdes v3.14.0 ${ }^{103}$ and collectively with MEGAHIT v1.2.9 104 411 (min k: 27, max k: 127, k step: 10). To generate corresponding coverage profiles for 412 assembled contigs, short reads were mapped back using Bowtie2 v2.3.5 ${ }^{105}$ with 413 default parameters. Subsequently, genome binning was performed using CONCOCT 414 v1.1.0 ${ }^{106}$, MaxBin2 v2.2. ${ }^{107}$, and MetaBAT2 v2.15 ${ }^{108}$ on contigs with length over 4152000 bp. Resulting bins from the same assembly were then dereplicated using 416 DAS_Tool v1.1.2 ${ }^{109}$. RefineM v0.0.25 ${ }^{110}$ was used to remove spurious contigs with 417 incongruent genomic and taxonomic properties. Applying a threshold average nucleotide identity of $99 \%$, bins from different assemblies were consolidated to a non-redundant set of metagenome-assembled genomes (MAGs) using dRep v2.5.4 111. Completeness and contamination of MAGs were assessed using CheckM v1.1.2 112. In total, 101 high quality (completeness > 90\% and contamination < 5\%) and 350 medium quality (completeness > 50\% and contamination < 10\%) ${ }^{44}$ MAGs from 18 phyla were recovered. Their corresponding taxonomy was assigned by GTDB-TK v1.3.0 ${ }^{113}$ with reference to GTDB R05-RS95 ${ }^{114}$. Open reading frames (ORFs) in MAGs were predicted using Prodigal v2.6.3 ${ }^{115}$.

\section{Community analysis}

428 Soil microbial community structures were determined by using both metagenomic and 16S rRNA gene amplicon sequencing. Community profiles in sequenced metagenomes were generated by mapping quality-filtered reads to the universal single copy ribosomal marker genes and clustering at $97 \%$ identity using SingleM v.0.12.1 (https://github.com/wwood/singlem). To align with the latest GTDB taxonomy at the time of submission (R05-RS95; release 2020/07), we generated a SingleM package for the single-copy ribosomal protein-encoding gene $r p / P$. In brief, 
437 downloaded. GraftM v0.12.2 ${ }^{116}$ was used to generate a phylogenetic package for

438 the sequences which was then used to make a community classification package by 439 SingleM v.0.12.1. For 16S rRNA gene amplicon sequencing, the DNeasy PowerSoil 440 kit (Qiagen) was used to extract DNA from $0.4 \mathrm{~g}$ of soil sample as per manufacturer's 441 instructions. The quality and concentration of DNA extracted were determined using 442 a Nanodrop spectrophotometer (ND-1000) and a Qubit Fluorometer. Quantitative 443 PCR (qPCR) using a 96-well plate in a pre-heated LightCycler 480 Instrument II 444 (Roche, Basel, Switzerland) was used to quantify the copy number of the 16S rRNA 445 genes in the samples as previously described ${ }^{117}$. For each sample, the V4 hypervariable region for 16S rRNA gene was amplified using the universal Earth Microbiome Project primer pairs F515 (Parada) ${ }^{118}$ and R806 (Apprill) ${ }^{119}$. Amplicons were sent to paired-end sequencing ( $2 \times 300 \mathrm{bp})$ on an Illumina MiSeq platform at the Australian Centre for Ecogenomics (ACE), University of Queensland. BBDuk function of the BBTools v38.80 was used to trim adapter sequences and filter PhiX contaminants as described above. The sequences were further processed on the QIIME2 platform (release 2019/07) ${ }^{120}$ to resolve amplicon sequence variants (ASVs)

453 through the following steps: (i) striping amplicons primers using cutadapt plugin ${ }^{121}$;

454 (ii) merging paired-end reads using q2-vsearch plugin ${ }^{122}$; (iii) quality filtering using a sliding window of four bases with an average Phred score 20; and (iv) de-noising and truncating sequences at 250 base pairs using deblur ${ }^{123}$. A total of 657,975 reads remained in the dataset (min: 13248, max: 102382) (Table S3). For taxonomic assignment, ASVs were independently annotated with trained naïve Bayes classifiers of $16 \mathrm{~S}$ rRNA reference databases Silva release $138^{124}$ and Greengenes $13.8^{125}$ (Table S3). Multiple sequence alignment of the sequences and subsequent phylogenetic tree building were performed using MAFFT ${ }^{126}$ and FastTree ${ }^{127}$, respectively, implemented in QIIME2. We then used $R$ packages phyloseq ${ }^{128}$, picante ${ }^{129}$, vegan ${ }^{130}$, betapart ${ }^{131}$ and ggplot2 ${ }^{132}$ for downstream statistical analysis and visualizations. Alpha diversity including observed richness, Chao1, Shannon index, and Faith's phylogenetic diversity were computed using estimate_richness function in phyloseq and pd function in picante. For beta diversity analysis, all samples were rarefied at the lowest sample sequencing depth, i.e. 13248 sequences per sample and rarefaction plots before and after rarefaction were shown in Figure 
multidimensional scaling ordination (NMDS) plot. To examine community turnover in relations to increasing geographic separation, a distance decay relationship of beta diversity (Bray-Curtis dissimilarity) against pairwise geographic distance was computed using the decay.model function fitted with a negative exponential law function in betapart. A $p$ value was calculated using the same function with 999 permutations (Table S3).

\section{Functional analysis}

To estimate the metabolic capability of the soil communities, metagenomes and derived genomes were searched against custom protein databases of representative metabolic marker genes using DIAMOND v.0.9.31 (query cover > 80\%) ${ }^{133}$. Searches were carried out using all quality-filtered unassembled reads with lengths over $140 \mathrm{bp}$ and the ORFs of the 451 MAGs. These genes are involved in sulfur cycling (AsrA, FCC, Sqr, DsrA, Sor, SoxB), nitrogen cycling (AmoA, HzsA, NifH, NarG, NapA, NirS, NirK, NrfA, NosZ, NxrA, NorB), iron cycling (Cyc2, MtrB, OmcB), reductive dehalogenation (RdhA), phototrophy (PsaA, PsbA, energy-converting microbial rhodopsin), methane cycling (McrA, MmoA, PmoA), hydrogen cycling (catalytic subunit of [NiFe]-hydrogenases, catalytic domain of [FeFe]-hydrogenases, and Fe-hydrogenases), isoprene oxidation (IsoA), carbon monoxide oxidation (CoxL, CooS), succinate oxidation (SdhA), fumarate reduction (FrdA), and carbon fixation (RbcL, AcsB, AclB, Mcr, HbsT, HbsC) ${ }^{48,52,134}$. Results were filtered based on an identity threshold of $50 \%$, except for group 4 [NiFe]-hydrogenases, [FeFe]hydrogenases, CoxL, AmoA, and NxrA (all 60\%), PsaA (80\%), PsbA and IsoA (70\%), and HbsT (75\%). Subgroup classification of reads was based on the closest match to the sequences in databases. To search for the presence of an additional set of genes involved in oxidative phosphorylation (AtpA), NADH oxidation (NuoF), aerobic respiration (CoxA, CcoN, CyoA, CydA), formate oxidation ( $\mathrm{FdhA}$ ), arsenic cycling (ARO, ArSC), and selenium cycling (YgfK), corresponding in-house databases were generated for this study. All archaeal and bacterial non-redundant proteins were retrieved from NCBI Refseq protein database release $99{ }^{135}$, which were then screened by hidden Markov models (HMM) ${ }^{136}$, with search cutoff scores as described previously ${ }^{137}$. Resulting hits were manually inspected to remove false positives and genes with lengths that deviated more than $20 \%$ from the average were discarded. The search of these genes in unassembled reads and ORFs of 
504 MAGs was carried out using the DIAMOND blastp algorithm with a minimum 505 percentage identity of $60 \%$ (NuoF), $70 \%$ (AtpA, ARO, YgfK) or $50 \%$ (all other 506 databases). Read counts for each gene were normalized to reads per kilobase per 507 million (RPKM) by dividing the actual read count by the total number of reads (in 508 millions) and then dividing by the gene length (in kilobases). In order to estimate the 509 gene abundance in the microbial community, high-quality unassembled reads were 510 also screened for the 14 universal single copy ribosomal marker genes used in 511 SingleM v.0.12.1 and PhyloSift ${ }^{138}$ by DIAMOND (query cover > 80\%, bitscore > 40) 512 and normalized as above. Subsequently, the average gene copy number of a gene 513 in the community was calculated by dividing the read count for the gene (in RPKM) 514 by the mean of the read counts of the 14 universal single copy ribosomal marker 515 genes (in RPKM).

\section{Phylogenetic analysis}

Maximum-likelihood phylogenetic trees were constructed to verify the presence and visualise the evolutionary history of key metabolic genes in the metagenomeassembled genomes and assembled unbinned reads. Trees were constructed using the amino acid sequences for subunits of ten enzymes involved in energy acquisition: group 1 [NiFe]-hydrogenase (HhyL, HylL); form I carbon monoxide dehydrogenase $(\mathrm{CoxL})$, particulate methane monooxygenase (PmoA), ammonia monooxygenase (AmoA), nitrite oxidoreductase (NxrA), sulfide-quinone oxidoreductase (Sqr), flavocytochrome $c$ sulfide dehydrogenase (FCC), thiosulfohydrolase (SoxB), iron-oxidizing c-type cytochrome (Cyc2), photosystem II $(\mathrm{PsbA})$, and energy-converting rhodopsins. Trees were also constructed of the amino acid sequences for subunits of three enzymes involved in carbon fixation: ribulose 1,5-bisphosphate carboxylase/oxygenase (RuBisCO; RbcL), thaumarchaeotal 4-hydroxybutyrate synthase ( $\mathrm{HbsT})$, and ATP-citrate lyase (AclB). In all cases, protein sequences retrieved from the MAGs or assembled metagenome sequences by homology-based searches were aligned against a subset of reference sequences from the custom protein databases using ClustalW ${ }^{139}$ in MEGA $X^{140}$. Evolutionary relationships were visualized by constructing maximum-likelihood phylogenetic trees; specifically, initial trees for the heuristic search were obtained automatically by applying Neighbour-Join and BioNJ algorithms to a matrix of pairwise distances estimated using a JTT model, and then selecting the topology 
538 with superior log likelihood value. All residues were used and trees were

539 bootstrapped with 50 replicates. To characterise the genetic context of [NiFe]

540 hydrogenases and ribulose-1,5-bisphosphate carboxylase / oxygenase (RuBisCO)

541 from the MAGs, up to 10 genes upstream and downstream of the catalytic subunits

542 were retrieved. These flanking genes were annotated against Pfam protein family

543 database v33.1 ${ }^{141}$ using PfamScan v1.6 ${ }^{142}$ and NCBI Refseq protein database

544 release $99{ }^{135}$ using DIAMOND ${ }^{133}$ blastp algorithm (default parameters). Alignments

545 with the highest score were retained and are summarised in Table S7. The $\mathrm{R}$

546 package gggenes (https://github.com/wilkox/gggenes) was used to construct gene

547 arrangement diagrams.

548

\section{Hydrogenase sequence analysis and homology modelling}

550 The amino acid sequence for the large (HylL; GBID = SMB94678) and small 551 subunits (HylS GBID = SMB94698) of the group 11 [NiFe]-hydrogenase from $H$. 552 roseosalivarius were inputted into the Phyre2 webserver using default parameters 143. The highest confidence output model for both subunits was derived from the 554 structure of the group $1 \mathrm{~h}$ [NiFe]-hydrogenase (HhyLS) from Cupriavidus necator H16 555 (PDB ID = 5AA5) ${ }^{71}$. The structure of the group 1 [NiFe]-hydrogenase tetramer was 556 assembled using Pymol, based on the tetrameric structure of the $C$. necator group 557 1h [NiFe]-hydrogenase for further analysis. To identify transmembrane helix 558 presence, position and topology in the HyITM proteins associated with group 11 559 [NiFe]-hydrogenases, the amino acid sequences from $H$. roseosalivarius were 560 inputted into the TMHHM 2.0 webserver $^{144}$.

\section{Gas chromatography assays}

563 Soil microcosms were used to determine the capacity of soil microbial communities 564 to oxidize $\mathrm{H}_{2}, \mathrm{CO}$, and $\mathrm{CH}_{4}$ by gas chromatography. For each of the 16 Mackay 565 Glacier region samples in technical duplicate, $2 \mathrm{~g}$ of soil was placed in a $120 \mathrm{ml}$ 566 serum vial and incubated at $10^{\circ} \mathrm{C}$. The ambient air headspace was amended with $567 \mathrm{H}_{2}, \mathrm{CO}$, and $\mathrm{CH}_{4}$ (via a mixed gas cylinder containing $0.1 \% \mathrm{v} / \mathrm{v} \mathrm{H}_{2}, \mathrm{CO}$, and $\mathrm{CH}_{4}$ 568 each in $\mathrm{N}_{2}$, BOC Australia) to give starting mixing ratios of approximately 10 parts 569 per million (ppmv) for each gas. At each time interval, $2 \mathrm{ml}$ of headspace gas was 570 sampled using a gas-tight syringe and stored in sealed a $3 \mathrm{ml}$ glass exetainer that 571 had been flushed with ultra-high purity $\mathrm{N}_{2}(99.999 \%$ pure, BOC Australia) prior to 
572 measurement. A VICl gas chromatographic machine with a pulsed discharge helium

573 ionization detector (model TGA-6791-W-4U-2, Valco Instruments Company Inc.) and

574 an autosampler was used to measure gas concentrations as previously described ${ }^{51}$.

575 The machine was calibrated against ultra-pure $\mathrm{H}_{2}, \mathrm{CO}$ and $\mathrm{CH}_{4}$ standards down to

576 the limit of quantification ( $\mathrm{H}_{2}: 20$ ppbv; $\mathrm{CO}: 9$ ppbv; $\mathrm{CH}_{4}: 500$ ppbv). Calibration

577 mixed gas (10.20 ppmv of $\mathrm{H}_{2}, 10.10$ ppmv of $\mathrm{CH}_{4}, 9.95$ ppmv of $\mathrm{CO}$ in $\mathrm{N}_{2}$, Air

578 Liquide Australia) and pressurized air (Air Liquide Australia) with known trace gas

579 concentrations were used as internal reference standards. Four pooled heat-killed

580 soils ( $2 \mathrm{~g}$ of pooled soil; treated at $121^{\circ} \mathrm{C}, 15$ p.s.i. for $60 \mathrm{mins}$ ) were prepared as

581 negative controls. For kinetic analysis, measurement time points with individual gas

582 concentration over 0.4 ppmv were used. First order reaction rate constants were

583 calculated by fitting an exponential model as determined by the lowest overall Akaike

584 information criterion value when compared to a linear model. Actual reaction rate

585 constants of the sample were obtained by correcting against means of negative

586 controls and only resultant values higher than the magnitude of measurement errors

587 of negative controls were retained. Bulk atmospheric gas oxidation rate for each

588 sample was calculated with respect to mean atmospheric mixing ratio of

589 corresponding trace gases $\left(\mathrm{H}_{2}: 0.53 \text { ppmv; CO: } 0.09 \text { ppmv; } \mathrm{CH}_{4}: 1.9 \mathrm{ppmv}\right)^{73,145,146}$.

590 Soil cell abundance was estimated using 16S rRNA gene copy number from qPCR

591 corrected with a reported average number of 16S rRNA gene copy per genome (i.e.

$5924.2)^{147}$. Cell specific gas oxidation rates were then inferred by dividing estimated soil

593 cell abundance and the proportion of corresponding gas oxidizers from metagenomic

594 data. To identify factors potentially influencing gas oxidation rates, a two-tailed all-vs-

595 all Spearman correlation matrix was generated that encompassed gas oxidation

596 rates, gas oxidation gene abundances, and soil physicochemical variables for each 


\section{References}

599

600

601

602

603

604

605

606

607

608

609

610

611

612

613

614

615

616

617

618

619

620

621

622

623

624

625

626

627

628

629

630

631

632

633

634

635

636

637

1. Leihy, R. I. et al. Antarctica's wilderness fails to capture continent's biodiversity. Nature 583, 567-571 (2020).

2. Cary, S. C., McDonald, I. R., Barrett, J. E. \& Cowan, D. A. On the rocks: the microbiology of Antarctic Dry Valley soils. Nat. Rev. Microbiol. 8, 129-138 (2010).

3. Convey, P. et al. The spatial structure of Antarctic biodiversity. Ecol. Monogr. 84, 203-244 (2014).

4. Cavicchioli, R. On the concept of a psychrophile. ISME J. 10, 793-795 (2016).

5. Chown, S. L. et al. The changing form of Antarctic biodiversity. Nature 522, 431-438 (2015).

6. Cowan, D. A., Russell, N. J., Mamais, A. \& Sheppard, D. M. Antarctic Dry Valley mineral soils contain unexpectedly high levels of microbial biomass. Extremophiles 6, 431-436 (2002).

7. Smith, J. J., Tow, L. A., Stafford, W., Cary, C. \& Cowan, D. A. Bacterial diversity in three different Antarctic Cold Desert mineral soils. Microb. Ecol. 51, 413-421 (2006).

8. Lee, C. K., Barbier, B. A., Bottos, E. M., McDonald, I. R. \& Cary, S. C. The Inter-Valley Soil Comparative Survey: the ecology of Dry Valley edaphic microbial communities. ISME J. 6, 1046-1057 (2012).

9. Ji, M. et al. Microbial diversity at Mitchell Peninsula, Eastern Antarctica: a potential biodiversity "hotspot". Polar Biol. 33, 237-249 (2015).

10. Lambrechts, S., Willems, A. \& Tahon, G. Uncovering the uncultivated majority in Antarctic soils: toward a synergistic approach. Front. Microbiol. 10, 242 (2019).

11. Pointing, S. B. et al. Highly specialized microbial diversity in hyper-arid polar desert. Proc. Natl. Acad. Sci. U. S. A. 107, 1254-1254 (2009).

12. Ji, M. et al. Atmospheric trace gases support primary production in Antarctic desert surface soil. Nature 552, 400-403 (2017).

13. Chan, Y., Van Nostrand, J. D., Zhou, J., Pointing, S. B. \& Farrell, R. L. Functional ecology of an Antarctic Dry Valley. Proc. Natl. Acad. Sci. U. S. A. 110, 8990-8995 (2013).

14. Peeters, K., Ertz, D. \& Willems, A. Culturable bacterial diversity at the Princess Elisabeth station (Utsteinen, Sør Rondane Mountains, East Antarctica) harbours many new taxa. Syst. Appl. Microbiol. 34, 360-367 (2011).

15. Pudasaini, S. et al. Microbial diversity of browning Peninsula, Eastern Antarctica revealed using molecular and cultivation methods. Front. Microbiol. 8, 591 (2017).

16. Tahon, G. \& Willems, A. Isolation and characterization of aerobic anoxygenic 
638

phototrophs from exposed soils from the Sør Rondane Mountains, East Antarctica. Syst. Appl. Microbiol. 40, 357-369 (2017).
17. Tahon, G., Tytgat, B., Lebbe, L., Carlier, A. \& Willems, A. Abditibacterium utsteinense sp. nov., the first cultivated member of candidate phylum FBP, isolated from ice-free Antarctic soil samples. Syst. Appl. Microbiol. 41, 279- 290 (2018).

18. Leung, P. M. et al. Energetic basis of microbial growth and persistence in desert ecosystems. mSystems 5, e00495-19 (2020).

19. Jones, S. E. \& Lennon, J. T. Dormancy contributes to the maintenance of microbial diversity. Proc. Natl. Acad. Sci. U. S. A. 107, 5881-5886 (2010).

20. Lennon, J. T. \& Jones, S. E. Microbial seed banks: the ecological and evolutionary implications of dormancy. Nat. Rev. Microbiol. 9, 119-130 (2011).

21. Rittershaus, E. S. C., Baek, S. H. \& Sassetti, C. M. The normalcy of dormancy: common themes in microbial quiescence. Cell Host and Microbe vol. 13 643651 (2013).

22. Hoehler, T. M. \& Jorgensen, B. B. Microbial life under extreme energy limitation. Nat. Rev. Microbiol. 11, 83-94 (2013).

23. Cockell, C. S. \& Stokes, M. D. Ecology: Widespread colonization by polar hypoliths. Nature 431, 414 (2004).

24. Bay, S., Ferrari, B. \& Greening, C. Life without water: How do bacteria generate biomass in desert ecosystems? Microbiol. Aust. 39, (2018).

25. Niederberger, T. D. et al. Carbon-fixation rates and associated microbial communities residing in arid and ephemerally Wet Antarctic Dry Valley soils. Front. Microbiol. 6, 1347 (2015).

26. Tahon, G., Tytgat, B., Stragier, P. \& Willems, A. Analysis of $c b b L$, nifH, and pufLM in Soils from the Sør Rondane Mountains, Antarctica, Reveals a Large Diversity of Autotrophic and Phototrophic Bacteria. Microb. Ecol. 71, 131-149 (2016).

27. Tahon, G., Tytgat, B. \& Willems, A. Diversity of key genes for carbon and nitrogen fixation in soils from the Sør Rondane Mountains, East Antarctica. Polar Biol. 41, 2181-2198 (2018).

28. Magalhães, C., Machado, A., Frank-Fahle, B., Lee, C. K. \& Cary, C. S. The ecological dichotomy of ammonia-oxidizing archaea and bacteria in the hyperarid soils of the Antarctic Dry Valleys. Front. Microbiol. 5, 515 (2014).

29. Tahon, G., Tytgat, B. \& Willems, A. Diversity of phototrophic genes suggests multiple bacteria may be able to exploit sunlight in exposed soils from the Sør Rondane Mountains, East Antarctica. Front. Microbiol. 7, 2026 (2016).

30. Edwards, C. R. et al. Draft genome sequence of uncultured upland soil cluster Gammaproteobacteria gives molecular insights into high-affinity methanotrophy. Genome Announc. 5, e00047-17 (2017).

31. Doran, P. T. et al. Antarctic climate cooling and terrestrial ecosystem 
response. Nature 415, 517-520 (2002).

32. Fountain, A. G., Nylen, T. H., Monaghan, A., Basagic, H. J. \& Bromwich, D. Snow in the McMurdo dry valleys, Antarctica. Int. J. Climatol. A J. R. Meteorol. Soc. 30, 633-642 (2010).

33. Van Goethem, M. W. et al. A reservoir of 'historical' antibiotic resistance genes in remote pristine Antarctic soils. Microbiome 6, 40 (2018).

34. Elberling, B. et al. Distribution and dynamics of soil organic matter in an Antarctic dry valley. Soil Biol. Biochem. 38, 3095-3106 (2006).

35. Adriaenssens, E. M. et al. Environmental drivers of viral community composition in Antarctic soils identified by viromics. Microbiome 5, 83 (2017).

36. Bay, S. K. et al. Soil bacterial communities exhibit strong biogeographic patterns at fine taxonomic resolution. mSystems 5, e00540-20 (2020).

37. Janssen, P. H. Identifying the dominant soil bacterial taxa in libraries of $16 \mathrm{~S}$ rRNA and 16S rRNA genes. Appl. Environ. Microbiol. 72, 1719-1728 (2006).

38. Delgado-Baquerizo, M. et al. A global atlas of the dominant bacteria found in soil. Science 359, 320-325 (2018).

39. Rotem, O., Pasternak, Z. \& Jurkevitch, E. The genus Bdellovibrio and like organisms. in The Prokaryotes: Deltaproteobacteria and Epsilonproteobacteria vol. 9783642390 3-17 (2014).

40. Collingro, A., Köstlbacher, S. \& Horn, M. Chlamydiae in the Environment. Trends Microbiol. 10.1016/j.tim.2020.05.020 (2020) doi:10.1016/j.tim.2020.05.020.

41. Yeoh, Y. K., Sekiguchi, Y., Parks, D. H. \& Hugenholtz, P. Comparative genomics of candidate phylum TM6 suggests that parasitism is widespread and ancestral in this lineage. Mol. Biol. Evol. 33, 915-927 (2016).

42. Brown, C. T. et al. Unusual biology across a group comprising more than $15 \%$ of domain Bacteria. Nature 523, 208-211 (2015).

43. Castelle, C. J. \& Banfield, J. F. Major new microbial groups expand diversity and alter our understanding of the tree of life. Cell 172, 1181-1197 (2018).

44. Bowers, R. M. et al. Minimum information about a single amplified genome (MISAG) and a metagenome-assembled genome (MIMAG) of bacteria and archaea. Nat. Biotechnol. 35, 725-731 (2017).

45. Constant, P., Chowdhury, S. P., Pratscher, J. \& Conrad, R. Streptomycetes contributing to atmospheric molecular hydrogen soil uptake are widespread and encode a putative high-affinity [NiFe]-hydrogenase. Environ. Microbiol. 12, 821-829 (2010).

46. Greening, C., Berney, M., Hards, K., Cook, G. M. \& Conrad, R. A soil actinobacterium scavenges atmospheric $\mathrm{H}_{2}$ using two membrane-associated, oxygen-dependent [NiFe] hydrogenases. Proc. Natl. Acad. Sci. U. S. A. 111, 4257-4261 (2014).

47. Greening, C. et al. Persistence of the dominant soil phylum Acidobacteria by 
trace gas scavenging. Proc. Natl. Acad. Sci. U. S. A. 112, 10497-10502 (2015).

48. Søndergaard, D., Pedersen, C. N. S. \& Greening, C. HydDB: a web tool for hydrogenase classification and analysis. Sci. Rep. 6, 34212 (2016).

49. Constant, P., Chowdhury, S. P., Hesse, L., Pratscher, J. \& Conrad, R. Genome data mining and soil survey for the novel Group 5 [NiFe]hydrogenase to explore the diversity and ecological importance of presumptive high-affinity $\mathrm{H}_{2}$-oxidizing bacteria. Appl. Environ. Microbiol. 77, 6027-6035 (2011).

50. King, G. M. Molecular and culture-based analyses of aerobic carbon monoxide oxidizer diversity. Appl. Environ. Microbiol. 69, 7257-7265 (2003).

51. Islam, Z. F. et al. Two Chloroflexi classes independently evolved the ability to persist on atmospheric hydrogen and carbon monoxide. ISME J. 13, 18011813 (2019).

52. Cordero, P. R. F. et al. Atmospheric carbon monoxide oxidation is a widespread mechanism supporting microbial survival. ISME J. 13, 2868-2881 (2019).

53. King, G. M. \& Weber, C. F. Distribution, diversity and ecology of aerobic COoxidizing bacteria. Nat. Rev. Microbiol. 5, 107-118 (2007).

54. Tabita, F. R. et al. Function, structure, and evolution of the RubisCO-like proteins and their RubisCO homologs. Microbiol. Mol. Biol. Rev. 71, 576-599 (2007).

55. Park, S. W. et al. Presence of duplicate genes encoding a phylogenetically new subgroup of form I ribulose 1,5-bisphosphate carboxylase/oxygenase in Mycobacterium sp. strain JC1 DSM 3803. Res. Microbiol. 160, 159-165 (2009).

56. Grostern, A. \& Alvarez-Cohen, L. RubisCO-based $\mathrm{CO}_{2}$ fixation and $\mathrm{C} 1$ metabolism in the actinobacterium Pseudonocardia dioxanivorans CB1190. Environ. Microbiol. 15, 3040-3053 (2013).

57. Greening, C., Villas-Bôas, S. G., Robson, J. R., Berney, M. \& Cook, G. M. The growth and survival of Mycobacterium smegmatis is enhanced by cometabolism of atmospheric $\mathrm{H}_{2}$. PLoS One 9, e103034 (2014).

58. Liot, Q. \& Constant, P. Breathing air to save energy - new insights into the ecophysiological role of high-affinity [NiFe]-hydrogenase in Streptomyces avermitilis. Microbiologyopen 5, 47-59 (2016).

59. Tveit, A. T. et al. Widespread soil bacterium that oxidizes atmospheric methane. Proc. Natl. Acad. Sci. U. S. A. 116, 8515-8524 (2019).

60. Islam, Z. F. et al. A widely distributed hydrogenase oxidises atmospheric $\mathrm{H} 2$ during bacterial growth. ISME J. 10.1038/s41396-020-0713-4 (2020) doi:10.1101/2020.04.14.040717.

61. Schmitz, R. A. et al. The thermoacidophilic methanotroph Methylacidiphilum fumariolicum SolV oxidizes subatmospheric $\mathrm{H}_{2}$ with a high-affinity, membrane- 
associated [NiFe] hydrogenase. ISME J. 14, 1223-1232 (2020).

62. Cordero, P. R. F. et al. Two uptake hydrogenases differentially interact with the aerobic respiratory chain during mycobacterial growth and persistence. J. Biol. Chem. 294, 18980-18991 (2019).

63. Lopes, A. R., Manaia, C. M. \& Nunes, O. C. Bacterial community variations in an alfalfa-rice rotation system revealed by $16 \mathrm{~S}$ rRNA gene 454pyrosequencing. FEMS Microbiol. Ecol. 87, 650-663 (2014).

64. Frindte, K., Pape, R., Werner, K., Löffler, J. \& Knief, C. Temperature and soil moisture control microbial community composition in an arctic-alpine ecosystem along elevational and micro-topographic gradients. ISME J. 13, 2031-2043 (2019).

65. Khilyas, I. V. et al. Microbial diversity and mineral composition of weathered serpentine rock of the Khalilovsky massif. PLoS One 14, e0225929 (2019).

66. Johnston, E. R. et al. Responses of tundra soil microbial communities to half a decade of experimental warming at two critical depths. Proc. Natl. Acad. Sci. 116, 15096-15105 (2019).

67. Whitman, W. B. Modest proposals to expand the type material for naming of prokaryotes. Int. J. Syst. Evol. Microbiol. 66, 2108-2112 (2016).

68. Murray, A. E. et al. Roadmap for naming uncultivated Archaea and Bacteria. Nat. Microbiol. 5, 987-994 (2020).

69. Hirsch, P. et al. Hymenobacter roseosalivarius gen. nov., sp. nov. from continental Antarctic soils and sandstone: bacteria of the Cytophaga/Flavobacterium/Bacteroides line of phylogenetic descent. Syst. Appl. Microbiol. 21, 374-383 (1998).

70. Schäfer, C., Friedrich, B. \& Lenz, O. Novel, oxygen-insensitive group 5 [NiFe]hydrogenase in Ralstonia eutropha. Appl. Environ. Microbiol. 79, 5137-45 (2013).

71. Schäfer, C. et al. Structure of an actinobacterial-type [NiFe]-hydrogenase reveals insight into $\mathrm{O}_{2}$-tolerant $\mathrm{H}_{2}$ oxidation. Structure 24, 285-292 (2016).

72. Ehhalt, D. H. \& Rohrer, F. The tropospheric cycle of $\mathrm{H}_{2}$ : a critical review. Tellus $B$ 61, 500-535 (2009).

73. Novelli, P. C., Masarie, K. A. \& Lang, P. M. Distributions and recent changes of carbon monoxide in the lower troposphere. J. Geophys. Res. Atmos. 103, 19015-19033 (1998).

74. Conrad, R. Soil microorganisms as controllers of atmospheric trace gases $\left(\mathrm{H}_{2}\right.$, $\mathrm{CO}, \mathrm{CH}_{4}$, OCS, $\mathrm{N}_{2} \mathrm{O}$, and NO). Microbiol. Mol. Biol. Rev. 60, 609-640 (1996).

75. Greening, C., Grinter, R. \& Chiri, E. Uncovering the metabolic strategies of the dormant microbial majority: towards integrative approaches. mSystems 4, e00107-19 (2019).

76. Tijhuis, L., Van Loosdrecht, M. C. \& Heijnen, J. J. A thermodynamically based correlation for maintenance gibbs energy requirements in aerobic and 

anaerobic chemotrophic growth. Biotechnol. Bioeng. 42, 509-519 (1993).

77. Price, P. B. \& Sowers, T. Temperature dependence of metabolic rates for microbial growth, maintenance, and survival. Proc. Natl. Acad. Sci. U. S. A. 101, 4631-4636 (2004).

78. LaRowe, D. E. \& Amend, J. P. Power limits for microbial life. Front. Microbiol. 6, 718 (2015).

79. Sundararaj, S. et al. The CyberCell Database (CCDB): A comprehensive, selfupdating, relational database to coordinate and facilitate in silico modeling of Escherichia coli. Nucleic Acids Res. 32, D293-D295 (2004).

80. Koch, A. L. What size should a bacterium be? A question of scale. Annu. Rev. Microbiol. 50, 317-348 (1996).

81. Finkel, O. M., Béja, O. \& Belkin, S. Global abundance of microbial rhodopsins. ISME J. 7, 448-451 (2013).

82. Pinhassi, J., DeLong, E. F., Béjà, O., González, J. M. \& Pedrós-Alió, C. Marine bacterial and archaeal ion-pumping rhodopsins: genetic Diversity, physiology, and ecology. Microbiol. Mol. Biol. Rev. 80, 929-954 (2016).

83. Gomez-Consarnau, L. et al. Proteorhodopsin Phototrophy Promotes Survival of Marine Bacteria during Starvation. Plos Biol. 8, (2010).

84. Steindler, L., Schwalbach, M. S., Smith, D. P., Chan, F. \& Giovannoni, S. J. Energy starved candidatus Pelagibacter ubique substitutes light-mediated ATP production for endogenous carbon respiration. PLoS One 6, e19725 (2011).

85. Panwar, P. et al. Influence of the polar light cycle on seasonal dynamics of an Antarctic lake microbial community. Microbiome 10.1186/s40168-020-00889-8 (2020).

86. Guerrero, L. D., Vikram, S., Makhalanyane, T. P. \& Cowan, D. A. Evidence of microbial rhodopsins in Antarctic Dry Valley edaphic systems. Environ.

Microbiol. 19, 3755-3767 (2017).

87. Oesterhelt, D. \& Stoeckenius, W. Rhodopsin-like protein from the purple membrane of Halobacterium halobium. Nat. new Biol. 233, 149-152 (1971).

88. Harris, A. et al. A new group of eubacterial light-driven retinal-binding proton pumps with an unusual cytoplasmic proton donor. Biochim. Biophys. Acta (BBA)-Bioenergetics 1847, 1518-1529 (2015).

89. Deeg, C. M. et al. Chromulinavorax destructans, a pathogen of microzooplankton that provides a window into the enigmatic candidate phylum Dependentiae. PLoS Pathog. 15, e1007801 (2019).

90. Beet, C. R. et al. Genetic diversity among populations of Antarctic springtails (Collembola) within the Mackay Glacier ecotone. Genome 59, 762-770 (2016).

91. Lambert, C. et al. Ankyrin-mediated self-protection during cell invasion by the bacterial predator Bdellovibrio bacteriovorus. Nat. Commun. 6, 1-10 (2015).

92. Pasternak, Z. et al. By their genes ye shall know them: genomic signatures of predatory bacteria. ISME J. 7, 756-769 (2013). 
93. Hamm, J. N. et al. Unexpected host dependency of Antarctic Nanohaloarchaeota. Proc. Natl. Acad. Sci. 116, 14661-14670 (2019).

94. Lagkouvardos, I. et al. Integrating metagenomic and amplicon databases to resolve the phylogenetic and ecological diversity of the Chlamydiae. ISME J. 8 , 115-125 (2014).

95. Jaffe, A. L., Castelle, C. J., Carnevali, P. B. M., Gribaldo, S. \& Banfield, J. F. The rise of diversity in metabolic platforms across the Candidate Phyla Radiation. BMC Biol. 18, 1-15 (2020).

96. Beam, J. P. et al. Ancestral absence of electron transport chains in Patescibacteria and DPANN. bioRxiv 2020.04.07.029462 (2020) doi:10.1101/2020.04.07.029462.

97. Cowan, D. A. \& Makhalanyane, T. P. Energy from thin air. Nature 552, 336337 (2017).

98. Lee, J. R. et al. Climate change drives expansion of Antarctic ice-free habitat. Nature 547, 49 (2017).

99. Rintoul, S. R. et al. Choosing the future of Antarctica. Nature 558, 233-241 (2018).

100. Cavicchioli, R. et al. Scientists' warning to humanity: microorganisms and climate change. Nat. Rev. Microbiol. 17, 569-586 (2019).

101. Kennicutt, M. C. 2nd et al. Six priorities for Antarctic science. Nature 512, 2325 (2014).

102. Heldmann, J. L. et al. The high elevation Dry Valleys in Antarctica as analog sites for subsurface ice on Mars. Planet. Space Sci. 85, 53-58 (2013).

103. Nurk, S., Meleshko, D., Korobeynikov, A. \& Pevzner, P. A. metaSPAdes: a new versatile metagenomic assembler. Genome Res. 27, 824-834 (2017).

104. Li, D. H. et al. MEGAHIT v1.0: A fast and scalable metagenome assembler driven by advanced methodologies and community practices. Methods 102, 311 (2016).

105. Langmead, B. \& Salzberg, S. L. Fast gapped-read alignment with Bowtie 2. Nat. Methods 9, 357 (2012).

106. Alneberg, J. et al. Binning metagenomic contigs by coverage and composition. Nat. Methods 11, 1144 (2014).

107. Wu, Y.-W., Simmons, B. A. \& Singer, S. W. MaxBin 2.0: an automated binning algorithm to recover genomes from multiple metagenomic datasets. Bioinformatics 32, 605-607 (2015).

108. Kang, D. et al. MetaBAT 2: an adaptive binning algorithm for robust and efficient genome reconstruction from metagenome assemblies. PeerJ 7, e7359 (2019).

109. Sieber, C. M. K. et al. Recovery of genomes from metagenomes via a dereplication, aggregation and scoring strategy. Nat. Microbiol. 1 (2018). 
110. Parks, D. H. et al. Recovery of nearly 8,000 metagenome-assembled genomes substantially expands the tree of life. Nat. Microbiol. 2, 1533 (2017).

111. Olm, M. R., Brown, C. T., Brooks, B. \& Banfield, J. F. dRep: a tool for fast and accurate genomic comparisons that enables improved genome recovery from metagenomes through de-replication. ISME J. 11, 2864 (2017).

112. Parks, D. H., Imelfort, M., Skennerton, C. T., Hugenholtz, P. \& Tyson, G. W. CheckM: assessing the quality of microbial genomes recovered from isolates, single cells, and metagenomes. Genome Res. 25, 1043-1055 (2015).

113. Chaumeil, P.-A., Mussig, A. J., Hugenholtz, P. \& Parks, D. H. GTDB-Tk: a toolkit to classify genomes with the Genome Taxonomy Database. Bioinformatics 36, 1925-1927 (2020).

114. Parks, D. H. et al. A standardized bacterial taxonomy based on genome phylogeny substantially revises the tree of life. Nat. Biotechnol. 36, 996-1004 (2018).

115. Hyatt, D. et al. Prodigal: prokaryotic gene recognition and translation initiation site identification. BMC Bioinformatics 11, 119 (2010).

116. Boyd, J. A., Woodcroft, B. J. \& Tyson, G. W. GraftM: a tool for scalable, phylogenetically informed classification of genes within metagenomes. Nucleic Acids Res. 46, e59-e59 (2018).

117. Chen, Y.-J. et al. Metabolic flexibility allows generalist bacteria to become dominant in a frequently disturbed ecosystem. bioRxiv 2020.02.12.945220 (2020).

118. Parada, A. E., Needham, D. M. \& Fuhrman, J. A. Every base matters: assessing small subunit $r R N A$ primers for marine microbiomes with mock communities, time series and global field samples. Environ. Microbiol. 18, 1403-1414 (2016).

119. Apprill, A., McNally, S., Parsons, R. \& Weber, L. Minor revision to V4 region SSU rRNA 806R gene primer greatly increases detection of SAR11 bacterioplankton. Aquat. Microb. Ecol. 75, 129-137 (2015).

120. Bolyen, E. et al. Reproducible, interactive, scalable and extensible microbiome data science using QIIME 2. Nat. Biotechnol. 37, 852-857 (2019).

121. Martin, M. Cutadapt removes adapter sequences from high-throughput sequencing reads. EMBnet.journal 17, 10 (2011).

122. Rognes, T., Flouri, T., Nichols, B., Quince, C. \& Mahé, F. VSEARCH: a versatile open source tool for metagenomics. PeerJ 4, 2584 (2016).

123. Amir, A. et al. Deblur rapidly resolves single-nucleotide community sequence patterns. mSystems 2, e00191-16 (2017).

124. Quast, C. et al. The SILVA ribosomal RNA gene database project: improved data processing and web-based tools. Nucleic Acids Res. 41, D590-D596 (2012).

125. McDonald, D. et al. An improved Greengenes taxonomy with explicit ranks for 
ecological and evolutionary analyses of bacteria and archaea. ISME J. 6, 610618 (2012).

126. Katoh, K., Misawa, K., Kuma, K. I. \& Miyata, T. MAFFT: A novel method for rapid multiple sequence alignment based on fast Fourier transform. Nucleic Acids Res. 30, 3059-3066 (2002).

127. Price, M. N., Dehal, P. S. \& Arkin, A. P. FastTree 2 - Approximately maximumlikelihood trees for large alignments. PLoS One 5, (2010).

128. McMurdie, P. J. \& Holmes, S. phyloseq: an R package for reproducible interactive analysis and graphics of microbiome census data. PLoS One 8, e61217 (2013).

129. Kembel, S. W. et al. Picante: R tools for integrating phylogenies and ecology. Bioinformatics 26, 1463-1464 (2010).

130. Dixon, P. VEGAN, a package of $R$ functions for community ecology. J. Veg. Sci. 14, 927-930 (2003).

131. Baselga, A. \& Orme, C. D. L. Betapart: An R package for the study of beta diversity. Methods Ecol. Evol. 3, 808-812 (2012).

132. Wickham, H. ggplot2: elegant graphics for data analysis. (Springer, 2016).

133. Buchfink, B., Xie, C. \& Huson, D. H. Fast and sensitive protein alignment using DIAMOND. Nat. Methods 12, 59 (2014).

134. Greening, C. et al. Diverse hydrogen production and consumption pathways influence methane production in ruminants. ISME J. 13, 2617-2632 (2019).

135. Pruitt, K. D., Tatusova, T. \& Maglott, D. R. NCBI reference sequences (RefSeq): a curated non-redundant sequence database of genomes, transcripts and proteins. Nucleic Acids Res. 35, D61-D65 (2007).

136. Eddy, S. R. Accelerated profile HMM searches. PLoS Comput. Biol. 7 , e1002195 (2011).

137. Anantharaman, K. et al. Thousands of microbial genomes shed light on interconnected biogeochemical processes in an aquifer system. Nat. Commun. 7, 13219 (2016).

138. Darling, A. E. et al. PhyloSift: phylogenetic analysis of genomes and metagenomes. PeerJ 2, e243 (2014).

139. Larkin, M. A. et al. Clustal $\mathrm{W}$ and Clustal $X$ version 2.0. Bioinformatics 23, 2947-2948 (2007).

140. Kumar, S., Stecher, G., Li, M., Knyaz, C. \& Tamura, K. MEGA X: molecular evolutionary genetics analysis across computing platforms. Mol. Biol. Evol. 35, 1547-1549 (2018).

141. Finn, R. D. et al. Pfam: The protein families database. Nucleic Acids Research vol. 42 D222-D230 (2014).

142. Madeira, F. et al. The EMBL-EBI search and sequence analysis tools APIs in 2019. Nucleic Acids Res. 47, W636-W641 (2019). 
965

966

967

968

969

970

971
143. Kelley, L. A., Mezulis, S., Yates, C. M., Wass, M. N. \& Sternberg, M. J. E. The Phyre2 web portal for protein modeling, prediction and analysis. Nat. Protoc. 10, 845-858 (2015).

144. Krogh, A., Larsson, B., Von Heijne, G. \& Sonnhammer, E. L. L. Predicting transmembrane protein topology with a hidden Markov model: Application to complete genomes. J. Mol. Biol. 305, 567-580 (2001).

145. Novelli, P. C. et al. Molecular hydrogen in the troposphere: global distribution and budget. J. Geophys. Res. Atmos. 104, 30427-30444 (1999).

146. Stocker, T. F. et al. Climate change 2013 the physical science basis: Working Group I contribution to the fifth assessment report of the intergovernmental panel on climate change. Climate Change 2013 the Physical Science Basis: Working Group I Contribution to the Fifth Assessment Report of the Intergovernmental Panel on Climate Change vol. 9781107057 (2013).

147. Větrovský, T. \& Baldrian, P. The variability of the $16 \mathrm{~S}$ rRNA gene in bacterial genomes and its consequences for bacterial community analyses. PLoS One 8, e57923 (2013).

148. Davis, K. E. R., Sangwan, P. \& Janssen, P. H. Acidobacteria, Rubrobacteridae and Chloroflexi are abundant among very slow-growing and mini-colonyforming soil bacteria. Environ. Microbiol. 13, 798-805 (2011). 


\section{Footnotes}

\section{Etymological information:}

988 Candidatus Edaphomicrobium (E.da.pho.mi.cro'bi.um. Gr. neut. n. edaphos, soil; 989 N.L. neut. n. microbium, a microbe; N. L. neut. n. Edaphomicrobium, a soil 990 microbium)

991 Candidatus Edaphomicrobium janssenii (jans.sen'i.i. N.L. gen. n. janssenii, of 992 Janssen, named after Peter $\mathrm{H}$. Janssen, for his pioneering isolation-based studies 993 that first described this lineage ${ }^{148}$ )

994 Candidatus Edaphomicrobiaceae (former candidate Chloroflexota family CSP1-4) 995 (E.da.pho.mi.cro.bi.a.ce'ae. N.L. neut. n. Edaphomicrobium a (Candidatus) bacterial 996 genus; suff. -aceae ending to denote a family; N.L. fem. pl. n. Edaphomicrobiaceae, 997 family of the genus Edaphomicrobium)

998 Candidatus Edaphomicrobiales (former candidate Chloroflexota order CSP1-4) 999 (E.da.pho.mi.cro.bi.a'les. N.L. neut. n. Edaphomicrobium a (Candidatus) bacterial 1000 genus; suff. -ales ending to denote an order; N.L. fem. pl. n. Edaphomicrobiales, 1001 order of the family Edaphomicrobiaceae)

1002 Candidatus Edaphomicrobia (former candidate Chloroflexota class Ellin6529) 1003 (E.da.pho.mi.cro'bi.a. N.L. neut. n. Edaphomicrobium a (Candidatus) bacterial 1004 genus; -ia ending to denote a class; N.L. neut. pl. n. Edaphomicrobia, class of the 1005 order Edaphomicrobiales)

1006

1007 Candidatus Aridivita (A.ri.di.vi'ta. L. masc. adj. aridus, dry; L. fem. n. vita, life; N.L. 1008 fem. n. Aridivita, a dry life)

1009 Candidatus Aridivita willemsiae (wil.lems'i.ae. N.L. gen. n. willemsiae, of Willems, 1010 named after Anne Willems, for her contributions to Antarctic microbiology using 1011 isolation-based approaches)

1012 Candidatus Aridivitaceae (A.ri.di.vi.ta.ce'ae. N.L. neut. n. Aridivita a (Candidatus) 1013 bacterial genus; suff. -aceae ending to denote a family; N.L. fem. pl. n. 1014 Aridivitaceae, family of the genus Aridivita)

1015 Candidatus Aridivitales (A.ri.di.vi.ta'les. N.L. neut. n. Aridivita a (Candidatus) 1016 bacterial genus; -ales ending to denote an order; N.L. fem. pl. n. Aridivitales, order 1017 of the family Aridivitaceae) 
1018 Candidatus Aridivitia (former candidate Actinobacteriota class UBA4738)

1019 (A.ri.di.vi'ti.a. N.L. neut. n. Aridivita a (Candidatus) bacterial genus; -ia ending to

1020 denote a class; N.L. neut. pl. n. Aridivitia, class of the order Aridivitales)

1021

1022 Data availability statement:

1023 All amplicon sequencing data, raw metagenomes, and metagenome-assembled 1024 genomes were deposited to the NCBI Sequence Read Archive under BioProject 1025 accession PRJNA630822.

1026

1027

\section{Acknowledgements:}

1028 This study was supported by an ARC DECRA Fellowship (DE170100310; awarded 1029 to C.G.), an Australian Antarctic Division grant (4592; awarded to C.G. and S.L.C.), a 1030 South African National Antarctic Program grant (110730: awarded to D.A.C), an 1031 NHMRC EL2 Fellowship (APP1178715; salary for C.G.), an Australian Government 1032 Research Training Stipend Scholarship and a Monash International Tuition 1033 Scholarship (awarded to P.M.L. and S.K.B.), and a National Research Foundation 1034 SANAP postdoctoral grant (awarded to M.O.). Logistic and financial support for the 1035 field work was provided by Antarctica New Zealand and the New Zealand Antarctic 1036 Research Institute, respectively (awarded to I.D.H.). We acknowledge the PhD 1037 student research discount offered by the Environmental Analysis Laboratory (EAL), a 1038 Southern Cross University NATA (National Association of Testing Authorities) 1039 ISO17025 accredited commercial and research support facility. We thank Thanavit 1040 Jirapanjawat for technical support, Maria Chuvochina for etymological advice, and 1041 Andrew Lovering, Philipp Nauer, Eleonora Chiri, Ricardo Cavicchioli, and Belinda 1042 Ferrari for helpful discussions.

1044 Author contributions:

1045 D.A.C., C.G., M.O., S.L.C., and I.D.H. conceived this study. C.G. and D.A.C. 1046 supervised this study. C.G., P.M.L., D.A.C., and M.O. designed experiments. P.M.L. 1047 and G.S. performed experiments. P.M.L., C.G., R.G., G.S., M.O., and D.A.C. 1048 analyzed data. P.M.L., C.G., R.G., D.A.C., M.O., and S.L.C. wrote the manuscript 1049 with input from all authors. Different authors were specifically responsible for the 1050 original sampling campaign (D.A.C., I.D.H.), metagenomic sequencing and assembly 1051 (P.M.L., C.G.), community analysis (P.M.L., G.S., C.G.), metabolic annotation 
1052 (P.M.L., C.G., M.O., D.A.C.), phylogenetic analysis (C.G., P.M.L., M.O., D.A.C., 1053 S.K.B.), genetic organization analysis (P.M.L., R.G., C.G., M.O., D.A.C.), molecular 1054 modelling (R.G., C.G.), biogeochemical analysis (P.M.L., C.G., G.S.), and 1055 physicochemical analysis (P.M.L., C.G.). K.J., S.V., M.V.G. and T.M. provided 1056 theoretical and logistical support.

1057

1058 The authors declare no conflict of interest. 
1059 Figures

1060

1061 Figure 1. Abundance, composition, and diversity of the microbial communities

1062 from the Mackay Glacier region. (a) Boxplot showing the estimated abundance of 1063 bacterial and archaeal taxa, based on 16S rRNA copy number determined by 1064 quantitative PCR. (b) Stacked bar chart showing phylum-level community 1065 composition based on metagenomic reads of the single-copy marker gene rp/P and 1066 metagenome-assembled genomes. Bacterial and archaeal taxonomy is based on 1067 Genome taxonomy database (GTDB) release 05-RS95. Phyla with less than 1\% 1068 abundance in the sample were grouped to "Other phyla". (c) Boxplot showing alpha 1069 diversity (Observed richness, Chao1, Shannon, Faith's phylogenetic diversity) of 1070 microbial communities based on 16S rRNA gene amplicon sequence variants. (d) 1071 Beta diversity of rarefied 16S rRNA gene amplicon sequencing data based on Bray1072 Curtis dissimilarity and visualised by a non-metric multidimensional scaling 1073 ordination (NMDS) plot.

Figure 2. Metabolic potential of the microbial communities to use inorganic 1076 compounds, organic compounds, and light for energy and carbon acquisition. Homology-based searches were used to identify signature genes encoding enzymes associated with (from top to bottom): oxidative phosphorylation, trace gas oxidation, sulfur compound oxidation, nitrification, other oxidative processes, photosynthesis, and carbon fixation. The left heatmap shows the percentage of total community members predicted to encode each signature metabolic gene. To infer abundance, read counts were normalized to gene length and the abundance of single-copy marker genes. The right heatmap shows the presence of these genes across the 451 metagenome-assembled genomes spanning 18 phyla. Abundance was normalized by predicted MAG completeness.

Figure 3. Identification of the novel group 11 family of [NiFe] hydrogenases identified in MAGs from this study. Amino acid sequences retrieved from the reconstructed genomes were aligned against reference sequences (bootstrapped 
1092 with 50 replicates). Branches of group 1 [NiFe] hydrogenases are shaded according

1093 to the subgroup classification and tips are colored based on phylum-level affiliation of 1094 the sequence. All sequences from MAGs of the Mackay Glacier region clustered with 1095 either the well-characterized group $1 \mathrm{~h}$ [NiFe]-hydrogenases or the previously 1096 unreported group 1 [ [NiFe]-hydrogenases. (b) Representative genetic organization of 1097 group 11 [NiFe] hydrogenase gene cluster derived from the Antarctic bacterium 1098 Hymenobacter roseosalivarius. This shows the predicted open reading frames for 1099 the large (HylL) and small (HylS) hydrogenase subunits, the five interposing short 1100 predicted transmembrane proteins (HylTM1-5), a predicted electron-relaying Rieske1101 type protein (HylE), and a maturation endopeptidase (HupD). Conserved open 1102 reading frames with no predicted function are shown but not labelled. (c) Three1103 dimensional model of the group 1 [ [NiFe] hydrogenase. This shows a structural 1104 homology model of a heterotetramer of HylL and HylS subunits as a ribbon 1105 representation and a cartoon of a speculative complex between the hydrogenase 1106 and genetically associated HylTM proteins. (d) The location of conserved residues 1107 coordinating the [NiFe]-centre of the HylL subunit and [FeS] clusters of the HylS 1108 subunit of the group 1 [ [NiFe] hydrogenase. (e) Putative location of [FeS] clusters 1109 and [NiFe] centre (spheres) in one half of the group 1 [ [NiFe] hydrogenase tetramer, 1110 with conserved coordinating residues (sticks) color coded as in panel C.

Figure 4. Rates of atmospheric trace gas oxidation by soils sampled from the 1113 Mackay Glacier region. Boxplots show rates of oxidation of atmospheric $\mathrm{H}_{2}, \mathrm{CO}$, 1114 and $\mathrm{CH}_{4}$ for each soil in duplicate soil microcosms at $10^{\circ} \mathrm{C}$, based on gas 1115 chromatography measurements. Only rates for samples with detectable gas 1116 oxidation are shown. (a) Atmospheric gas oxidation rate for each microcosm 1117 normalized to wet weight of soil. (b) Cell-specific reaction rates for each microcosm. 1118 These rates were calculated by dividing the estimated soil cell abundance and 1119 proportion of gas oxidizers based on quantitative qPCR and metagenome short read 1120 analysis (HhyL and HylL abundance for $\mathrm{H}_{2}$, CoxL abundance for CO, PmoA and 1121 MmoX abundance for $\mathrm{CH}_{4}$ ). 
a

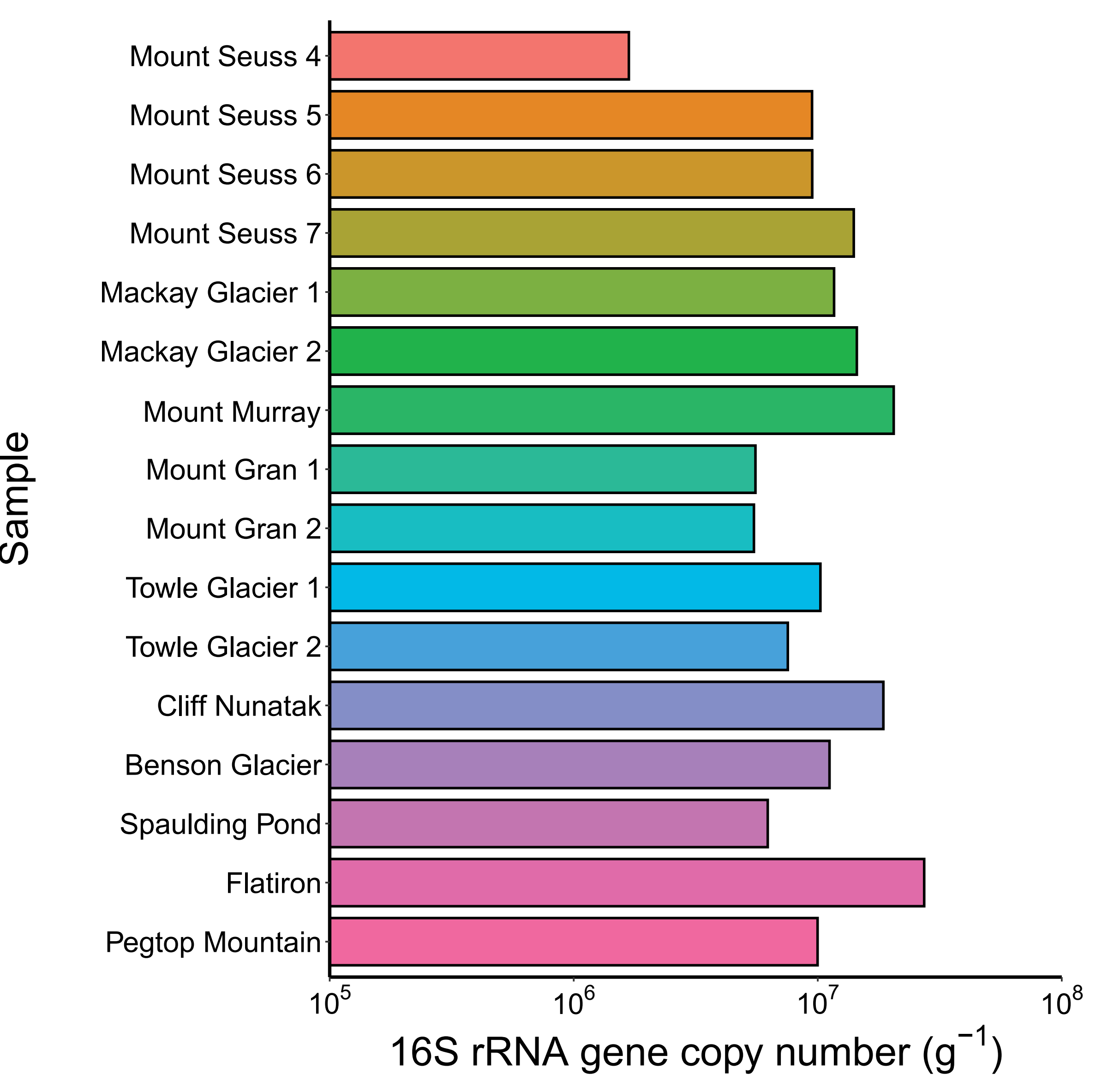

b
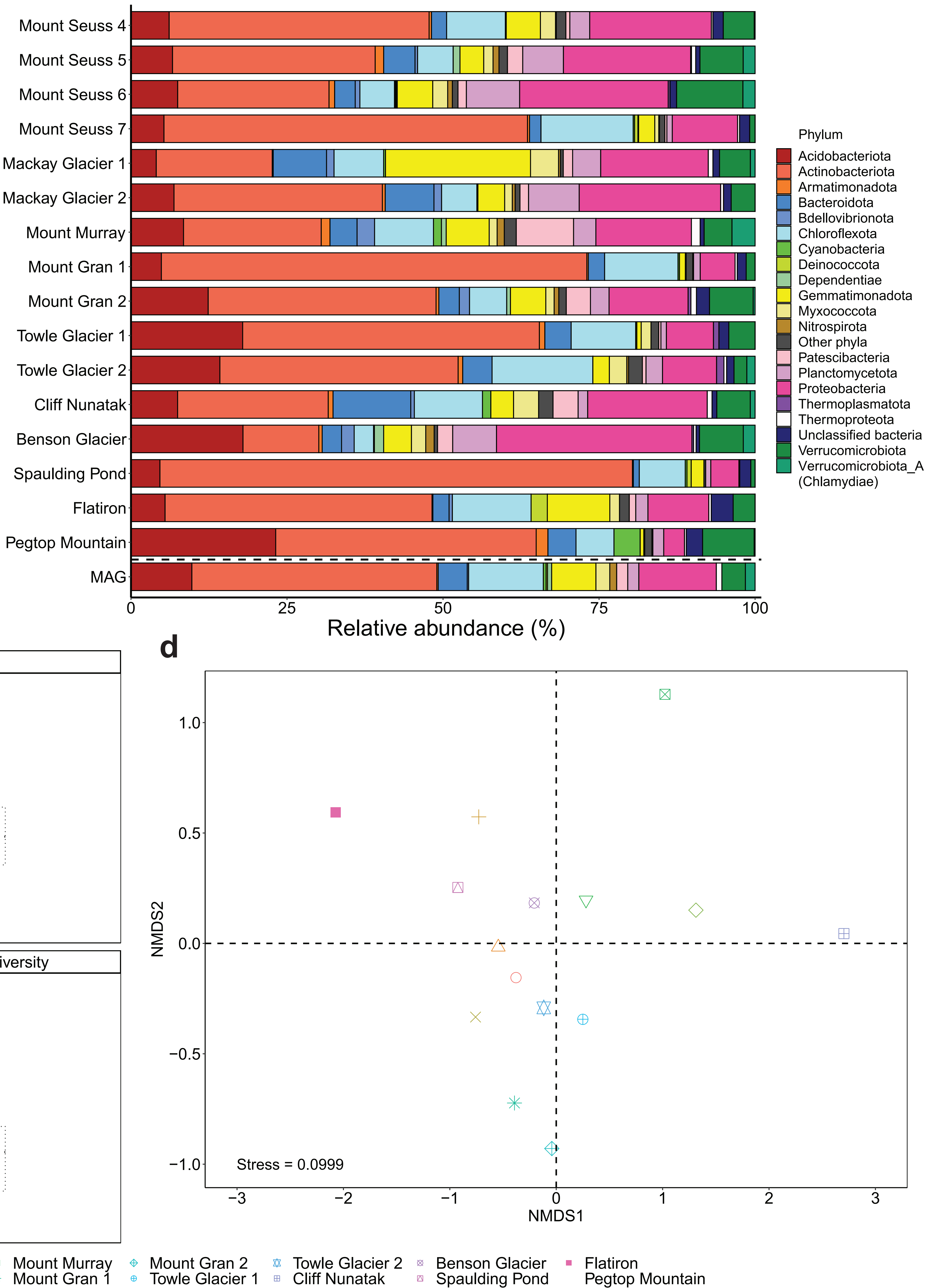

Towle Glacier 1 - Cliff Nunatak 2 B Spaulding Pond $₫$ Pegtop Mountain

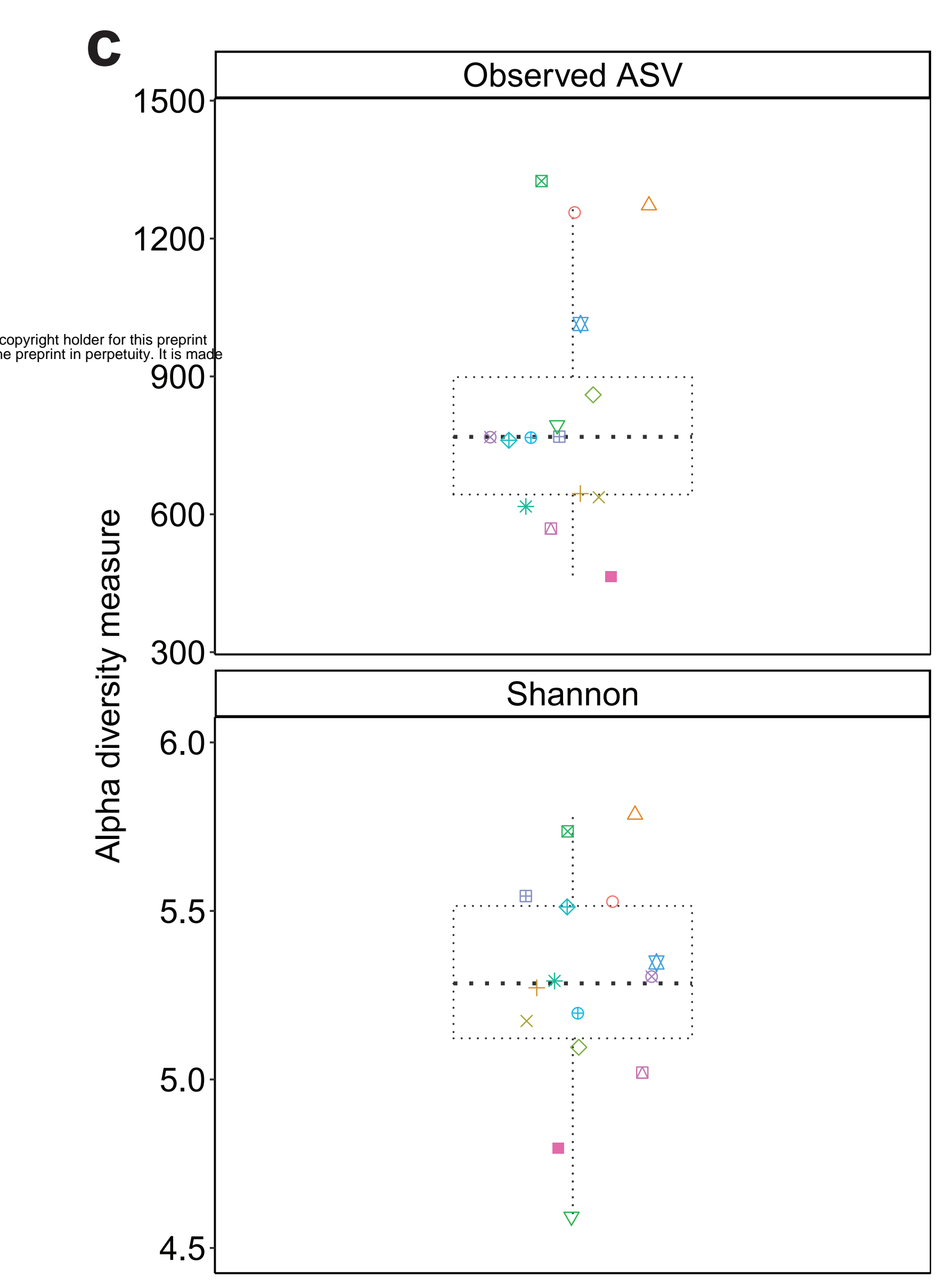

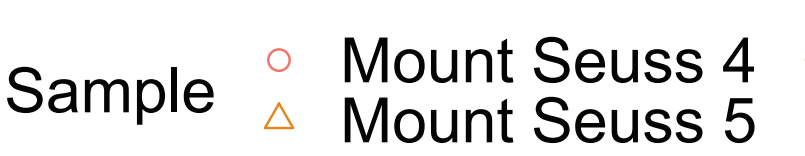

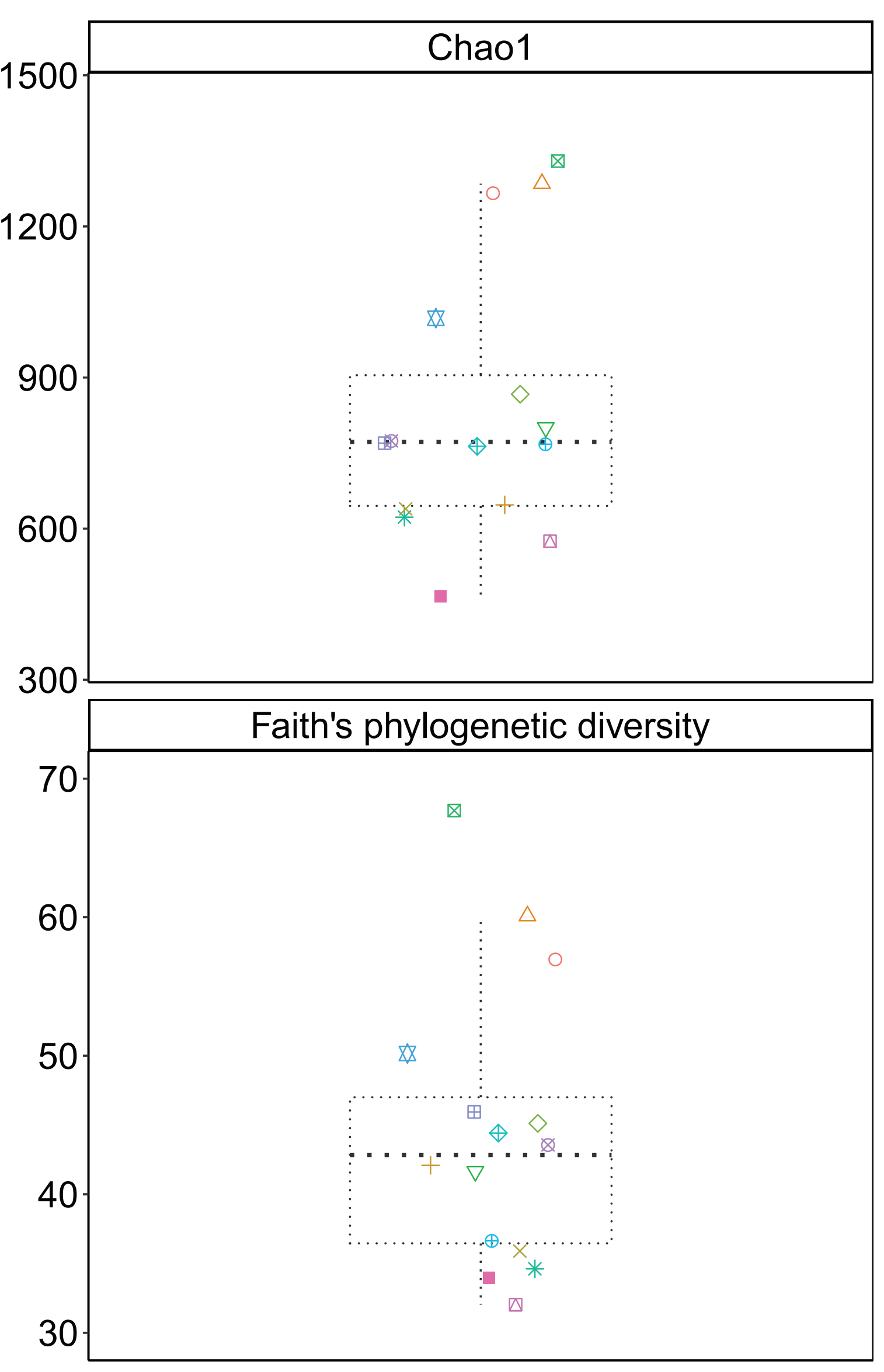

Mount Seuss $6 \diamond$ Mackay Glacier $1 \otimes$ Mount Murray 


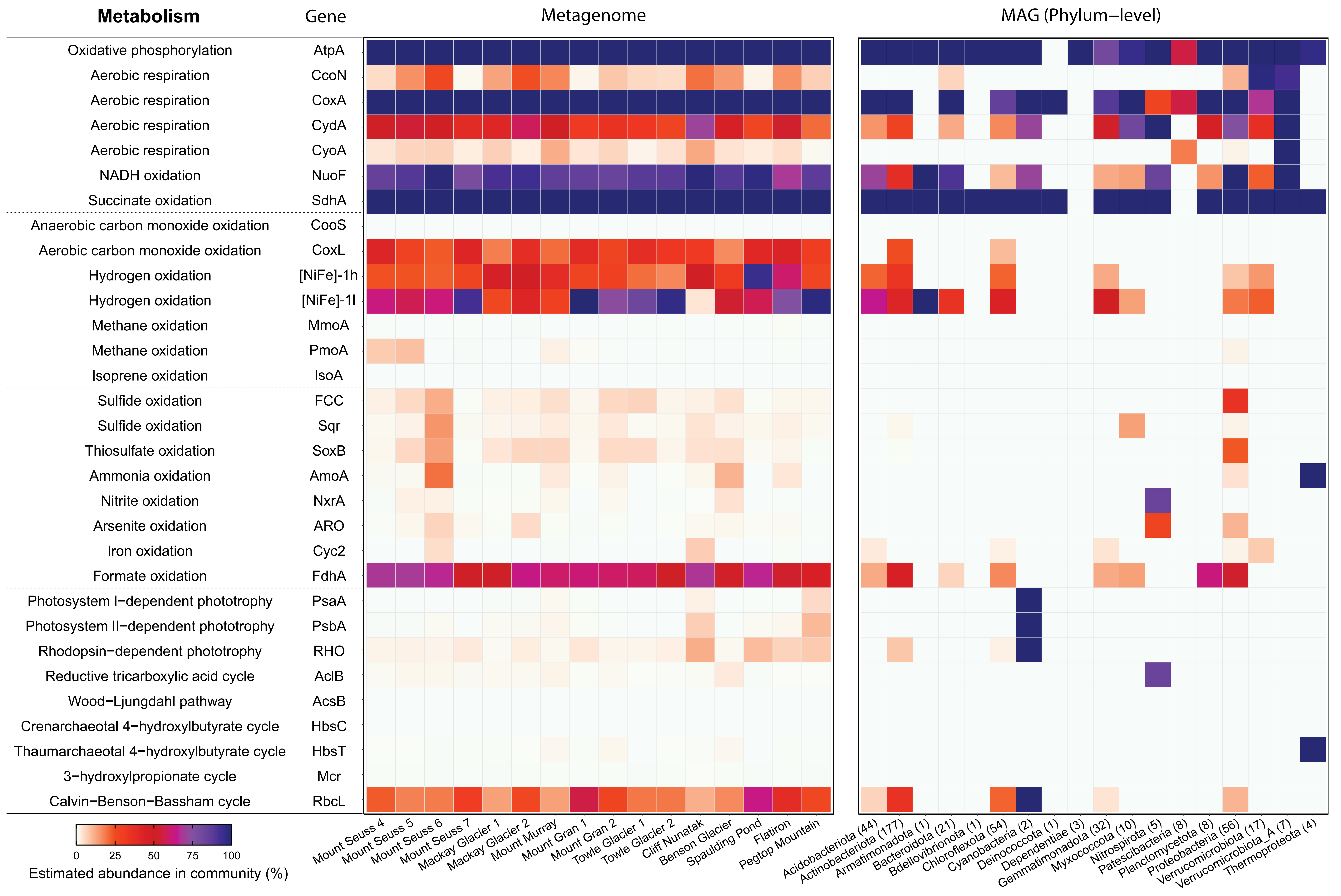




\section{a}

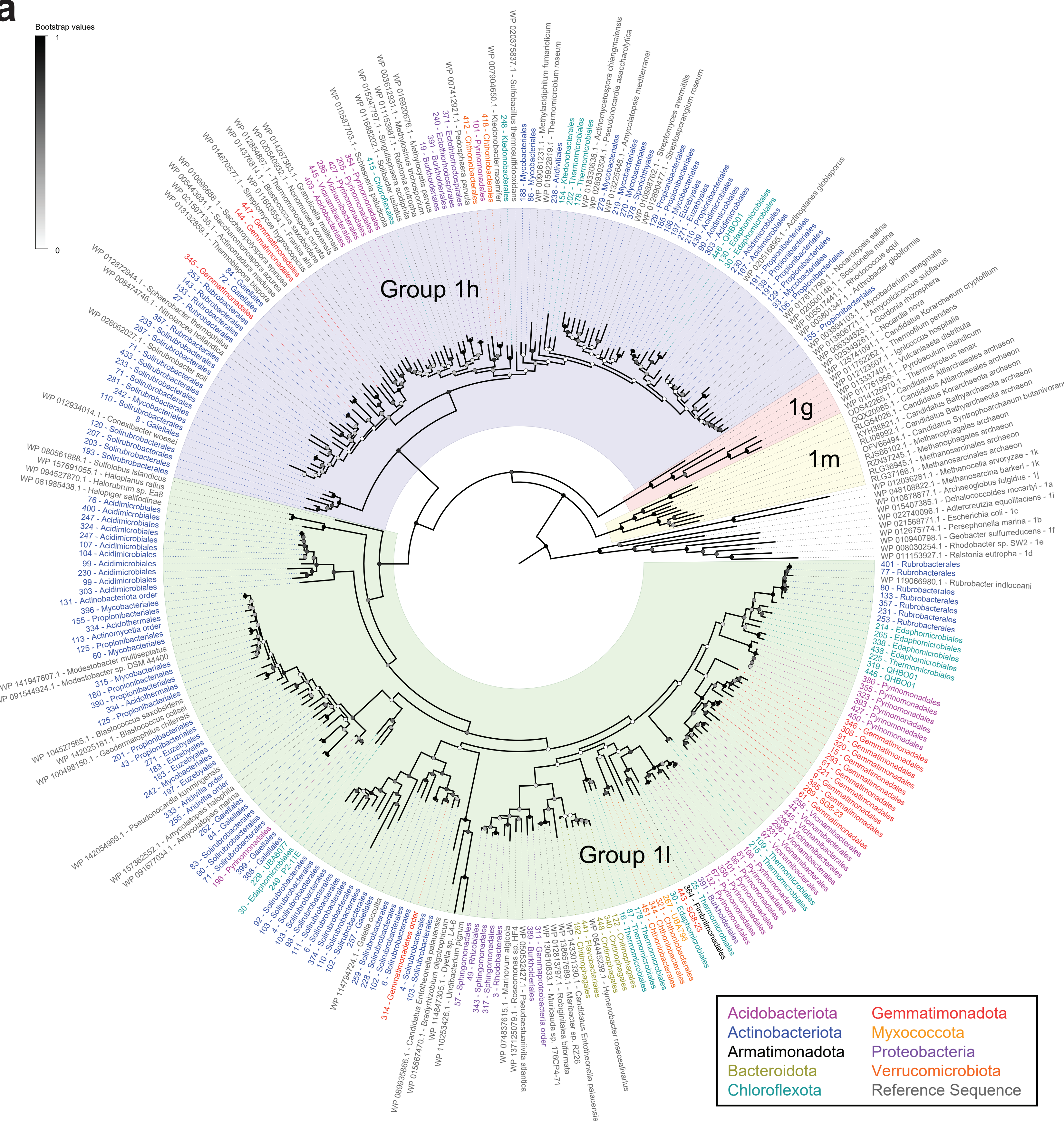

b

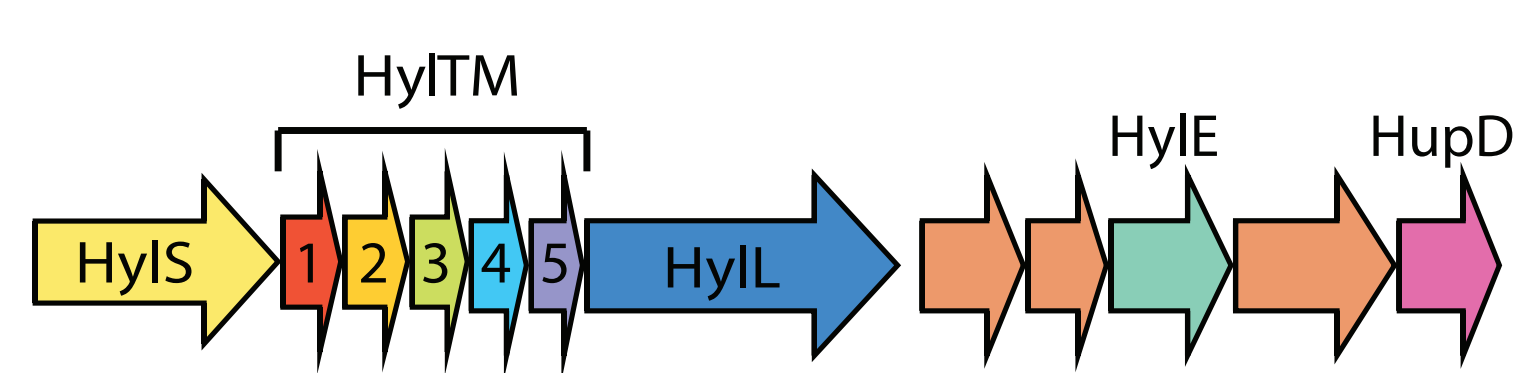

C

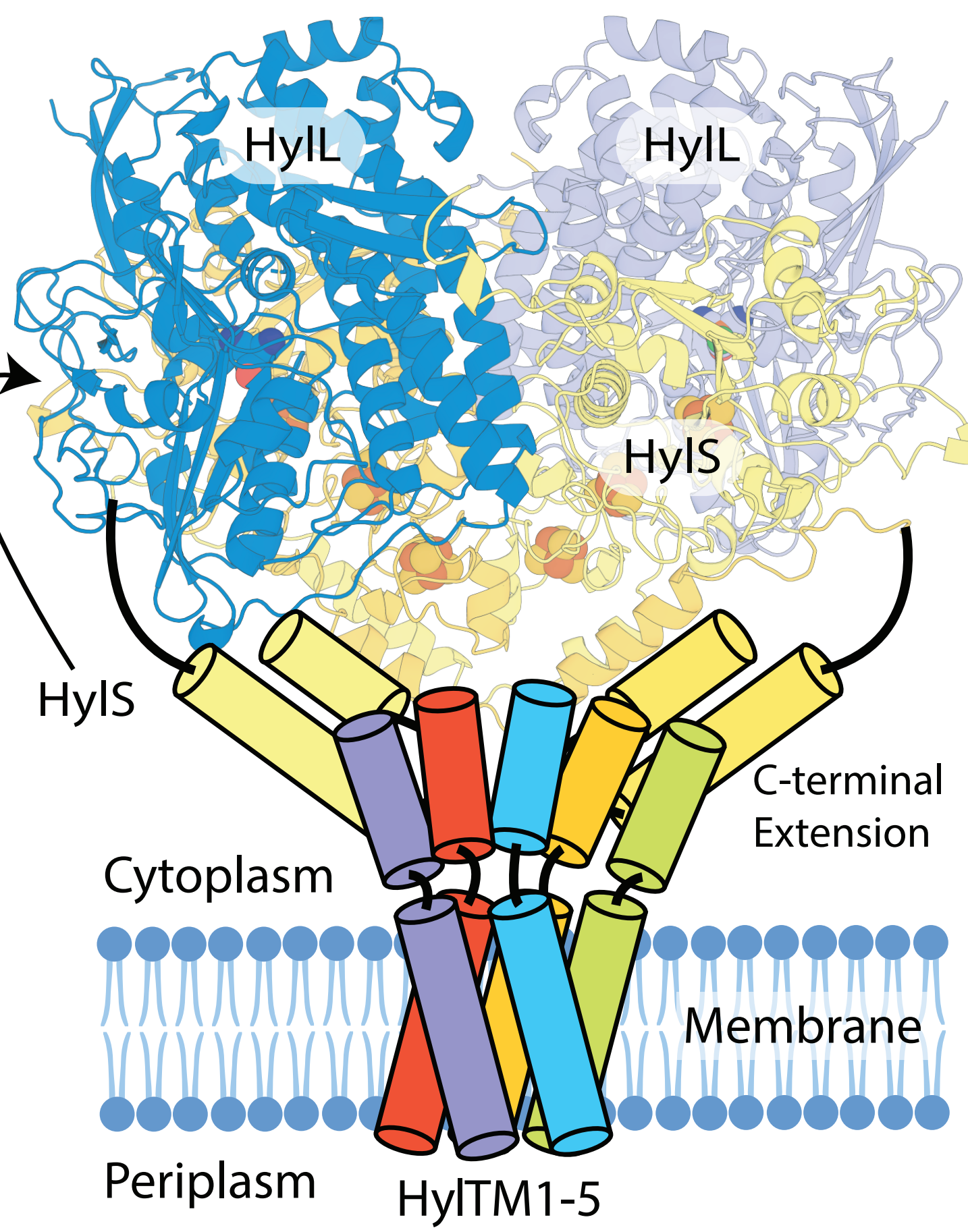

d
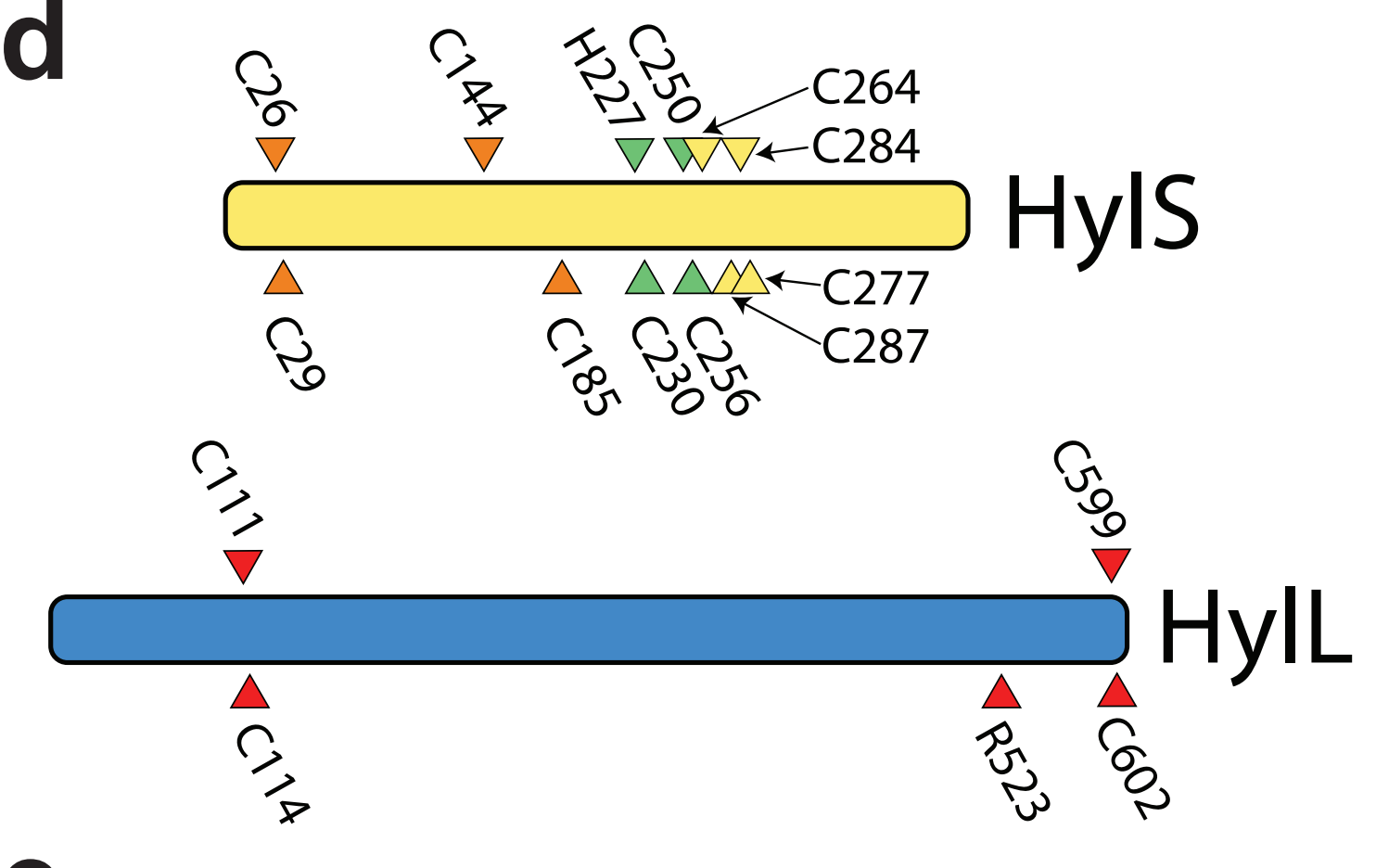

e

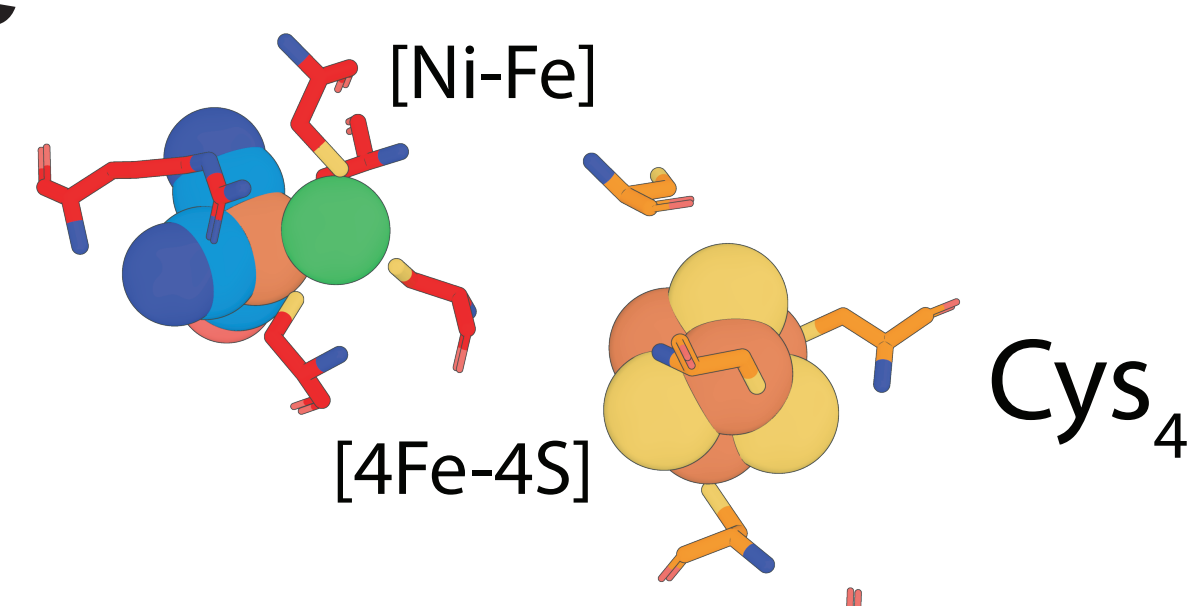

[4Fe-4S] $\int_{\pi} \mathrm{Cys}_{4}$

[4Fe-4S] $\mathrm{Cys}_{3} \mathrm{His}$ 
a
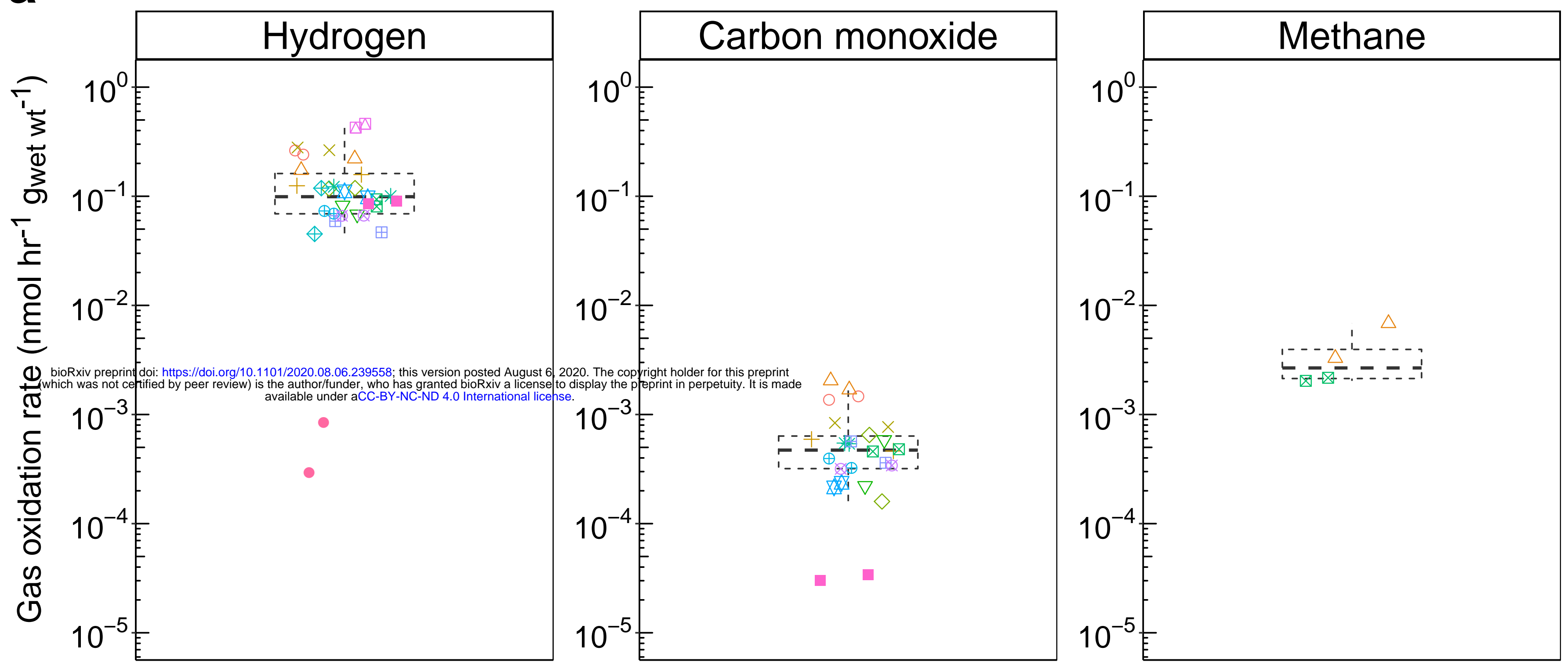

b
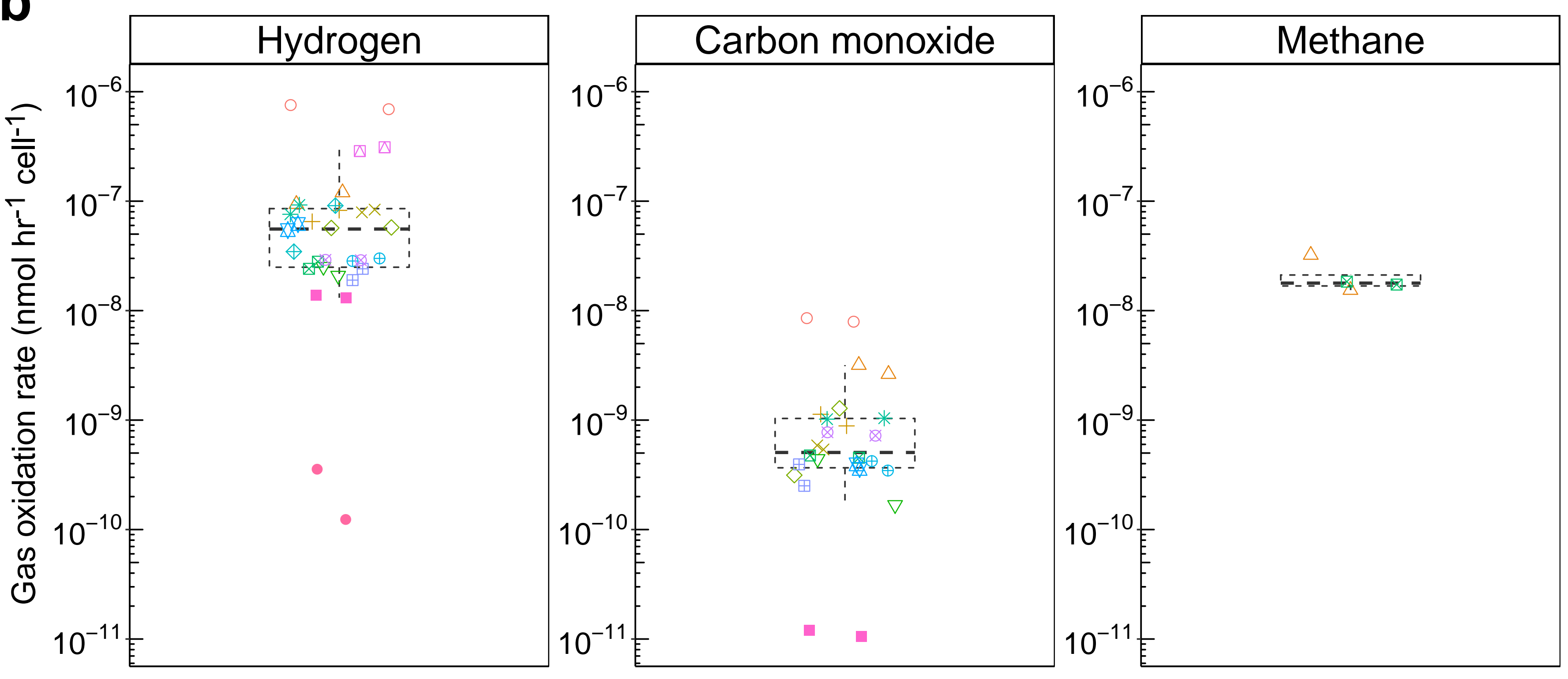

Sample

Mount Seuss 4 Mount Seuss 5 Mount Seuss 6 Mount Seuss 7
Mackay Glacier $1 \bowtie$ Mount Gran 2 Mackay Glacier 2 Mount Murray Mount Gran 1
Towle Glacier 1 Towle Glacier 2 Cliff Nunatak
Benson Glacier Spaulding Pond Flatiron

- Pegtop Mountain 